\title{
A topology optimization method based on the level set method incorporating a fictitious interface energy
}

\section{$\operatorname{AUTHOR}(S):$}

Yamada, Takayuki; Izui, Kazuhiro; Nishiwaki, Shinji; Takezawa, Akihiro

\section{CITATION:}

Yamada, Takayuki ...[et al]. A topology optimization method based on the level set method incorporating a fictitious interface energy. Computer Methods in Applied Mechanics and Engineering 2010, 199(45-48): 2876-2891

\section{ISSUE DATE:}

2010-11-15

URL:

http://hdl.handle.net/2433/131866

\section{RIGHT:}

(c) 2010 Elsevier B.V.; この論文は出版社版でありません。引用の際には 出版社版をご確認ご利用ください。; This is not the published version. Please cite only the published version. 


\title{
A Topology Optimization Method Based on the Level Set Method Incorporating a Fictitious Interface Energy
}

\author{
Takayuki Yamada ${ }^{\text {a,*,** }}$ Kazuhiro Izui ${ }^{\text {a }}$ Shinji Nishiwaki ${ }^{a}$ \\ Akihiro Takezawa ${ }^{\mathrm{b}}$ \\ ${ }^{a}$ Graduate School of Engineering, Kyoto University, Yoshida-honmachi, Sakyo-ku, \\ Kyoto, 606-8501, JAPAN \\ ${ }^{\mathrm{b}}$ Graduate School of Engineering, Hiroshima University, 1-4-1 Kagamiyama, \\ Higashi-Hiroshima, Hiroshima, 739-8527, JAPAN
}

\begin{abstract}
This paper proposes a new topology optimization method, which can adjust the geometrical complexity of optimal configurations, using the level set method and incorporating a fictitious interface energy derived from the phase field method. First, a topology optimization problem is formulated based on the level set method, and the method of regularizing the optimization problem by introducing fictitious interface energy is explained. Next, the reaction-diffusion equation that updates the level set function is derived and an optimization algorithm is then constructed, which uses the Finite Element Method to solve the equilibrium equations and the reaction-diffusion equation when updating the level set function. Finally, several optimum design examples are shown to confirm the validity and utility of the proposed topology optimization method.
\end{abstract}

Key words: Topology Optimization, Finite Element Method, Level Set Method, Phase Field Method, Tikhonov Regularization Method

\section{Introduction}

This paper proposes a new level set-based topology optimization method, which can control the geometrical complexity of obtained optimal configurations, using a fictitious interface energy based on the concept of the phase

\footnotetext{
* Corresponding author. Tel:+81 75753 5198; fax:+81 757535857

**Email adresses: takayuki-yamada@astro.mbox.media.kyoto-u.ac.jp
} 
field model [1-4]. The novel aspect of the proposed method is the incorporation of level set-based boundary expressions and fictitious interface energy in the topology optimization problem, and the replacement of the original topology optimization problem with a procedure to solve a reaction-diffusion equation.

Structural optimization has been successfully used in many industries such as automotive industries. Structural optimization can be classified into sizing[5,6], shape[7-11] and topology optimization[12-14], the last offering the most potential for exploring ideal and optimized structures. Topology optimization has been extensively applied to a variety of structural optimization problems such as the stiffness maximization problem $[12,15]$, vibration problems [16-18], optimum design problems for compliant mechanisms [19,20], and thermal problems [21-23], after Bensdøe and Kikuchi [12] first proposed the so-called Homogenization Design Method. The basic concepts of topology optimization are (1) the extension of a design domain to a fixed design domain, and (2) replacement of the optimization problem with material distribution problem, using the characteristic function [24]. A homogenization method [12,25$28]$ is utilized to deal with the extreme discontinuity of material distribution and to provide the material properties viewed in a global sense as homogenized properties. The Homogenization Design Method (HDM) has been applied to a variety of design problems. The density approach [29], also called the SIMP (Solid Isotropic Material with Penalization) method [30,31], is another currently used topology optimization method, the basic idea of which is the use of a fictitious isotropic material whose elasticity tensor is assumed to be a function of penalized material density, represented by an exponent parameter. Bendsøe and Sigmund [32] asserted the validity of the SIMP method in view of the mechanics of composite materials. The phase field model based on the theory of phase transitions [1-4] is also used as another approach toward regularizing topology optimization problems and penalizing material density [33-38]. In addition to the above conventional approaches, a different type of method, called the evolutionary structural optimization (ESO) method [18,39], has been proposed. In this method, the design domain is discretized using a finite element mesh and unnecessary elements are removed based on heuristic criteria so that the optimal configuration is ultimately obtained as an optimal subset of finite elements.

Unfortunately, the conventional topology optimization methods tend to suffer from numerical instability problems $[40,41]$, such as mesh dependency, checkerboard patterns and grayscales. Several methods have been proposed to mitigate these instability problems, such as the use of high-order finite elements [40] and filtering schemes [41]. Although various filtering schemes are currently used, they crucially depend on artificial parameters that lack rational guidelines for determining appropriate a priori parameter values. Additionally, optimal configurations can include highly complex geometrical structures 
that are inappropriate from an engineering and manufacturing standpoint. Although a number of geometrical constraint methods for topology optimization methods have been proposed, such as the perimeter control method [42] and member size control method [43,44], the parameters and the complexity of obtained optimal configurations are not uniquely linked. Furthermore, geometrical constraint methods often make the optimization procedure unstable. Thus, a geometric constraint method in which the complexity of the optimal configuration can be set uniquely, and which also maintains stability in the optimization procedure, has yet to be proposed.

A different approach is used in level set-based structural optimization methods that have been proposed as a new type of structural optimization method. Such methods implicitly represent target structural configurations using the iso-surface of the level set function, which is a scalar function, and the outlines of target structures are changed by updating the level set function during the optimization process. The level set method was originally proposed by Osher and Sethian [45] as a versatile method to implicitly represent evolutional interfaces in an Eulerian coordinate system. The evolution of the boundaries with respect to time is tracked by solving the so-called Hamilton-Jacobi partial differential equation, with an appropriate normal velocity that is the moving boundary velocity normal to the interface. Level set methods are potentially useful in a variety of applications, including fluid mechanics [46-48], phase transitions [49], image processing [50-52] and solid modeling in CAD [53].

In level set-based structural optimization methods, complex shape and topological changes can be handled and the obtained optimal structures are free from grayscales, since the structural boundaries are represented as the isosurface of the level set function. Although these relatively new structural optimization methods overcome the problems of checkerboard patterns and grayscales, mesh dependencies have yet to be eliminated.

Sethian and Wiegmann [54] first proposed a level set-based structural optimization method where the level set function is updated using an ad hoc method based on the Von Mises stress. Osher and Santosa [55] proposed a structural optimization method where the shape sensitivity is used as the normal velocity, and the structural optimization is performed by solving the level set equation using the upwind scheme. This proposed method was applied to eigen-frequency problems for an inhomogeneous drum using a two-phase optimization of the membrane where the mass density assumes two different values, while the elasticity tensor is constant over the entire domain.

Belytschko et al. [56] proposed a topology optimization using an implicit function to represent structural boundaries and their method allows topological changes by introducing the concept of an active zone where the material properties such as Young's modulus are smoothly distributed. Wang et al. [57] 
proposed a shape optimization method based on the level set method where the level set function is updated using the Hamilton-Jacobi equation, also called the level set equation, based on the shape sensitivities and the proposed method was applied to the minimum mean compliance problem. Wang and Wang [58] extended this method to a multi-material optimal design problem using a "color" level set method where $m$ level set functions are used to represent $2^{m}$ different material phases. Luo et al. [59] and Chen et al. [60] proposed a level set-based shape optimization method that controls the geometric width of structural components using a quadratic energy functional based on image active contour techniques. Allaire et al. [61] independently proposed a level set-based shape optimization method where the level set function is updated using smoothed shape sensitivities that are mapped to the design domain using a smoothing technique. A simple "ersatz material" approach was employed to compute the displacement field of the structure, and optimal configurations were obtained for the minimum compliance problem for both structures composed of linear elastic and non-linear hyperelastic material, and compliant mechanism structural design problems. Allaire and Jouve [62] also extended their method to lowest eigen-frequency maximization problems and minimum compliance problems having multiple loads. Recently, numerous extensions of the level set-based method have been presented, such as the use of different expressions [63], the use of a specific numerical method such as meshless methods [64], the use of mathematical approaches in the optimization scheme [65], and other applications [66-70].

The above level set-based structural optimization methods can be said to be a type of shape optimization method, since the shape boundaries of target structures are evolved from an initial configuration by updating the level set equation using shape sensitivities. Therefore, topological changes that increase the number of holes in the material domain are not permitted, although topological changes that decrease the number of holes are allowed. As a result, the obtained optimal configurations strongly depend on the given initial configuration. Rong and Liang [71] and Yamada et al. [72] pointed out that in level set-based structural optimization using the Hamilton-Jacobi equation, the movement of the structural boundaries stops at the boundaries of the fixed design domain because the level set function has a non-zero value there, and as a result, inappropriate optimal configurations are obtained. To provide for the possibility of topological changes, Allaire et al. [73] introduced the bubble method [74] to a level set-based shape optimization method using topological derivatives [75-77]. In Allaire's method [73], structural boundaries are updated based on smoothed shape sensitivities using the level set equation and holes are introduced during the optimization process. Appropriate optimal configurations were obtained using several different initial configurations, however parameter setting with respect to the introduction of holes during the optimization process was difficult and potentially affected the obtained optimal configurations. 
Wang et al. [78] proposed an extended level set method for a topology optimization method based on one of their previously proposed methods [57]. In their method [78], an extended velocity which has a non zero value in the material domain is introduced and the level set function is not reinitialized to maintain the property of a signed distance function. Topological changes including the introduction of holes in a material domain are therefore allowed, however the extended velocity cannot be logically determined, since the level set equation is derived based the boundary advection concept. As a result, it is difficult to define appropriate extended velocities and the definition of the extension velocities in large measure determines the shape of the obtained optimal structures.

In level set-based shape optimization methods using the Hamilton-Jacobi equation, the level set function must be re-initialized to maintain the signed distance characteristic of the function. This re-initialization operation [79-81] is not an easy task, and a number of level set-based topology optimization methods that do not depend on boundary advection concepts have been proposed recently. Wei and Wang $[65,66]$ proposed a piecewise constant level set method used in their topology optimization method. In this method, an objective functional is formulated as the sum of a primary objective functional and a structural perimeter, which regularizes the optimization problem. However, obtained optimal configurations can differ dramatically depending on the initial configuration, since the setting of certain parameters of the constraint functional for the piecewise constant level set function greatly affects the updating of the level set function.

In this research, we propose a topology optimization method using a level set model incorporating a fictitious interface energy derived from the phase field concept, to overcome the numerical problems mentioned above. The proposed method, a type of topology optimization method, also has the advantage of allowing not only shape but also topological changes. In addition, the proposed method allows the geometrical complexity of the optimal configuration to be qualitatively specified, a feature resembling the perimeter control method, and does not require re-initialization operations during the optimization procedure. In the following sections, a topology optimization problem is formulated based on the level set method, and the method of regularizing the optimization problem by introducing a fictitious interface energy is explained. The reaction-diffusion equation that updates the level set function is then derived. Here, we use the ersatz material approach to compute the equilibrium equations of the structure on an Eulerian coordinate system. The proposed topology optimization method is then applied to the minimum mean compliance problem, the optimum design problem of compliant mechanisms and the lowest eigenfrequency problem. Next, an optimization algorithm for the proposed method is constructed using the Finite Element Method. Finally, to confirm the validity and utility of the proposed topology optimization method, 
several numerical examples are provided for both two- and three-dimensional cases.

\section{Formulations}

\subsection{Topology optimization method}

Consider a structural optimization problem that determines the optimal configuration of a domain filled with a solid material, i.e., a material domain $\Omega$ that denotes the design domain, by minimizing an objective functional $F$ under a constraint functional $G$ concerning the volume constraint, described as follows:

$$
\begin{array}{ll}
\inf _{\Omega} & F(\Omega)=\int_{\Omega} f(\mathbf{x}) \mathrm{d} \Omega \\
\text { subject to } & G(\Omega)=\int_{\Omega} \mathrm{d} \Omega-V_{\max } \leq 0,
\end{array}
$$

where $V_{\max }$ is the upper limit of the volume constraint and $\mathbf{x}$ represents a point located in $\Omega$. In conventional topology optimization methods [12], a fixed design domain $D$, composed of a material domain $\Omega$ such that $\Omega \subset D$, and another complementary domain representing a void exists, i.e., a void domain $D \backslash \Omega$ is introduced. Using the characteristic function $\chi_{\Omega} \in L^{\infty}$ defined as

$$
\chi_{\Omega}(\mathbf{x})=\left\{\begin{array}{lll}
1 & \text { if } & \mathbf{x} \in \Omega \\
0 & \text { if } & \mathbf{x} \in D \backslash \Omega,
\end{array}\right.
$$

the above structural optimization problem is replaced by a material distribution problem, to search for an optimal configuration of the design domain in the fixed design domain $D$ as follows:

$$
\begin{array}{ll}
\inf _{\chi_{\Omega}} & F\left(\chi_{\Omega}(\mathbf{x})\right)=\int_{D} f(\mathbf{x}) \chi_{\Omega}(\mathbf{x}) \mathrm{d} \Omega \\
\text { subject to } \quad G\left(\chi_{\Omega}(\mathbf{x})\right)=\int_{D} \chi_{\Omega}(\mathbf{x}) \mathrm{d} \Omega-V_{\max } \leq 0 .
\end{array}
$$

In the above formulation, topological changes as well as shape change are allowed during the optimization procedure.

However, it is commonly accepted that topology optimization problems are ill-posed because the obtained configurations expressed by the characteristic function can be very discontinuous. That is, since the characteristic function $\chi$ is defined as a subset of a bounded Lebesgue space $L^{\infty}$ which is only assured integrability, the obtained solutions may be discontinuous anywhere in the fixed 
design domain. To overcome this problem, the design domain is relaxed using various regularization techniques such as the homogenization method [26-28]. In the homogenization method, microstructures that represent the composite material status are introduced. In two-scale modeling, microstructures are continuously distributed almost everywhere in the fixed design domain $D$. The regularized and sufficiently continuous physical properties are obtained as the homogenized properties. Burger and Stainko [38], Wang and Zhou [33,37] and Zhou and Wang [34,35] proposed an alternative regularization method using the Tikhonov regularization method [82]. In these methods, by adding a Cahn-Hilliard-type penalization functional [1] to an objective functional, the topology optimization problem is regularized and the material density penalized. The phase field model utilized in certain structural optimization methods employs a regularization technique based on the imposition of some degree of shape smoothness, but these methods also yield optimal configurations that include grayscales.

In these regularization techniques, the existence of grayscales is allowed in the obtained optimal configurations. Although such grayscales can be interpreted as being micro-porous in the physical sense, they are problematic in the engineering sense since such obtained optimal solutions are difficult to interpret as practical designs that can be manufactured. Furthermore, the optimal configurations obtained by conventional topology optimization methods can include highly complex structures that are also inappropriate from an engineering and manufacturing standpoint. To mitigate these problems, a method using a perimeter constraint [42] and methods using a density gradient constraint $[43,44]$ have been proposed. In the former, however, the obtained results crucially depend on artificial parameters that require appropriate, but elusive, values to obtain desired results. And in the latter, use of the density gradient constraint increases grayscales. Also, methods employing perimeter or density gradient constraints are poor at adjusting the geometrical complexity of the obtained optimal configurations, since the relation of the geometrical complexity of the configuration and the optimization parameters cannot be uniquely determined. Hitherto, a method that allows the geometrical complexity of obtained optimal structures to be manipulated has not been proposed.

On the other hand, level set-based structural optimization methods have been proposed $[45,57,61]$. In these methods, the level set function $\phi(\mathbf{x})$ is introduced to represent a boundary $\partial \Omega$ between the material and void domains. That is, the boundary is expressed using the level set function $\phi(\mathbf{x})$ as follows:

$$
\left\{\begin{array}{lll}
\phi(\mathbf{x})>0 & \text { for } & \forall \mathbf{x} \in \Omega \backslash \partial \Omega \\
\phi(\mathbf{x})=0 & \text { for } & \forall \mathbf{x} \in \partial \Omega \\
\phi(\mathbf{x})<0 & \text { for } & \forall \mathbf{x} \in D \backslash \Omega .
\end{array}\right.
$$

Using the above level set function, an arbitrary topology as well as shape of 
the material domain $\Omega$ in domain $D$ can be implicitly represented, and level set boundary expressions are free of grayscales. In level set-based methods, the evolution of the boundaries with respect to fictitious times is tracked by solving the so-called Hamilton-Jacobi partial differential equation (explained below), with an appropriate normal velocity that is the velocity of the moving boundary normal to the interface. However, as Allarie et al. [61] discussed, this problem is basically ill-posed, and in order to regularize the structural optimization problems, certain smoothness, geometrical, or topological constraint, such as a perimeter constraint [85] must be imposed. Furthermore, topological changes that increase the number of holes in the material domain may not occur, although topological changes that decrease the number of holes are allowed. As a result, the obtained optimal configurations strongly depends on the given initial configuration.

In this research, to overcome the above major problems in the conventional topology optimization methods and level set-based structural optimization methods, we propose a new level set-based topology optimization method using a fictitious interface energy based on the phase field model.

In the proposed approach, first, the definition of the level set function is modified per the following equation to introduce the fictitious interface energy in the phase field model to regularize the topology optimization problem:

$$
\left\{\begin{array}{lll}
1 \geq \phi(\mathbf{x})>0 & \text { for } & \forall \mathbf{x} \in \Omega \backslash \partial \Omega \\
\phi(\mathbf{x})=0 & \text { for } & \forall \mathbf{x} \in \partial \Omega \\
0>\phi(\mathbf{x}) \geq-1 & \text { for } & \forall \mathbf{x} \in D \backslash \Omega
\end{array}\right.
$$

We assume that the distribution of the level set function $\phi$ must have the same property of distribution as the phase field variable in the phase field method. Based on this assumption, the level set function $\phi$ has upper and lower limit constraints imposed in Equation (7). In addition, in sufficiently distant regions from the structural boundaries, the value of the level set function must be equivalent to 1 or -1 .

Here, by adding a fictitious interface energy term derived from the concept of the phase field model to the objective functional, the regularized topology optimization problem is described using the relaxed characteristic function that is a function of the level set function, defined as follows:

$$
\begin{array}{ll}
\inf _{\phi} & F_{R}\left(\chi_{\phi}(\phi), \phi\right)=\int_{D} f(\mathbf{x}) \chi_{\phi}(\phi) \mathrm{d} \Omega+\int_{D} \frac{1}{2} \tau|\nabla \phi|^{2} \mathrm{~d} \Omega \\
\text { subject to } & G\left(\chi_{\phi}(\phi)\right)=\int_{D} \chi_{\phi}(\phi) \mathrm{d} \Omega-V_{\max } \leq 0,
\end{array}
$$

where $F_{R}$ is a regularized objective functional and $\chi_{\phi}(\phi) \in L^{2}$ is a sufficiently smooth characteristic function, since the level set function $\phi$ is assumed to be 
continuous and is formulated as

$$
\Phi=\left\{\phi(\mathbf{x}) \mid \phi(\mathbf{x}) \in H^{1}(D)\right\}
$$

As a result, the former optimization problem is replaced with a problem to minimize the energy functional, which is the sum of the objective functional and the fictitious interface energy, where $\tau>0$ is a regularization parameter representing the ratio of the fictitious interface energy and the objective functional.

Note that the fictitious interface energy term here is equivalent to the so-called Chan-Hilliard energy, and it plays a very important role in regularizing the optimization problem. By introducing this term, the optimization problem is sufficiently relaxed and the obtained optimal configurations have sufficient smoothness. The optimization problem also becomes numerically stable. It is well-known that the Chan-Hilliard energy converges exactly to the perimeter. As a result, our optimal configurations are obtained under an implicitly imposed geometrical constraint. This regularization is called the Tikhonov regularization method, and details concerning its theoretical background are available in the literature $[82,83]$. It is possible to control the degree of complexity of obtained optimal structures by adjusting the value of the coefficient of regularization $\tau$. Strictly speaking, the regularization technique employed here is a perimeter constraint method, just as regularization techniques applied to the original topology optimization method implicitly impose geometric constraints. We note that Leitao and Scherzer [84] proposed a shape optimization method incorporating the Tikhonov regularization method and level set method, however the basic concept of their method differs from ours, which is a topology optimization method.

Next, the optimization problem represented by (8) and (9) is reformulated using Lagrange's method of undetermined multipliers. Let the Lagrangian be $\bar{F}$ and the Lagrange multiplier of the volume constraint be $\lambda$. The optimization problem is then formulated as

$$
\begin{aligned}
\inf _{\phi} \bar{F}_{R}\left(\chi_{\phi}(\phi), \phi\right)= & \int_{D} f(\mathbf{x}) \chi_{\phi}(\phi) \mathrm{d} \Omega \\
& +\lambda\left(\int_{D} \chi_{\phi}(\phi) \mathrm{d} \Omega-V_{\max }\right)+\int_{D} \frac{1}{2} \tau|\nabla \phi|^{2} \mathrm{~d} \Omega \\
= & \int_{D} \bar{f}(\mathbf{x}) \chi_{\phi}(\phi) \mathrm{d} \Omega-\lambda V_{\max }+\int_{D} \frac{1}{2} \tau|\nabla \phi|^{2} \mathrm{~d} \Omega,
\end{aligned}
$$

where the density function of the Lagrangian $\bar{f}(\mathbf{x})$ is such that $\bar{f}(\mathbf{x})=f(\mathbf{x})+\lambda$. The optimal configuration will be obtained by solving the above optimization problem.

Next, the necessary optimality conditions (KKT-conditions) for the above 
optimization problem are derived as follows:

$$
\left\langle\frac{\mathrm{d} \bar{F}_{R}\left(\chi_{\phi}(\phi), \phi\right)}{\mathrm{d} \phi}, \Phi\right\rangle=0, \quad \lambda G\left(\chi_{\phi}(\phi)\right)=0, \quad \lambda \geq 0, \quad G\left(\chi_{\phi}(\phi)\right) \leq 0
$$

where the notation $\left\langle\frac{\mathrm{d} \bar{F}_{R}\left(\chi_{\phi}(\phi), \phi\right)}{\mathrm{d} \phi}, \Phi\right\rangle$ represents the Fréchet derivative of the regularized Lagrangian $\bar{F}_{R}$ with respect to $\phi$ in the direction of $\Phi$. The level set function describing the optimal configurations satisfies the above KKT conditions. Conversely, solutions obtained by Equation (13) are optimal solution candidates, but obtaining this level set function directly is problematic. Here, the optimization problem is replaced by a problem of solving time evolutional equations, which will provide optimal solution candidates.

\subsection{The time evolutional equations}

Let a fictitious time $t$ be introduced, and assume that the level set function $\phi$ is also implicitly a function of $t$, to represent structural changes in the material domain $\Omega$ over time. In past level set-based structural optimization method research [57][61], the outline of target structures is updated by solving the following time evolutional equation:

$$
\frac{\partial \phi(\mathbf{x}, t)}{\partial t}+V_{N}(\mathbf{x}, t)|\nabla \phi(\mathbf{x}, t)|=0 \quad \text { in } D
$$

where $V_{N}(\mathbf{x}, t)$ is the normal velocity function, which is given as a smoothed shape derivative of material domain $\Omega$ since the above equation represents shape changes during fictitious optimization process times. Therefore, level set-based structural optimization methods using Equation (14) are essentially shape optimization methods. That is, only the shape boundary of the material domain evolves during the optimization process, and topological changes that generate holes in the material domain do not occur. As a result, the initial configuration settings profoundly affect the obtained optimal configuration.

To provide for the possibility of topological changes, Allaire et al. [73] proposed a method for introducing holes using topological derivatives [75-77], a concept that is basically the same as the bubble method [74] where the optimal position at which a hole is to be introduced is analytically derived. However, in Allaire's method, the obtained optimal structure depends on the setting of various parameters and it can be difficult to stably obtain optimal structures. Especially in problems where heat conduction and structural configuration are coupled, or static electric field, heat conduction and structural configuration are coupled, we encountered situations where convergence was poor and stably obtained optimal structures were elusive [70]. 
A new update method is developed in this research to replace the use Equation (14). Here, we assume that variation of the level set function $\phi(t)$ with respect to fictitious time $t$ is proportional to the gradient of the Lagrangian $\bar{F}$, as shown in the following:

$$
\frac{\partial \phi}{\partial t}=-K(\phi) \frac{\mathrm{d} \bar{F}_{R}}{\mathrm{~d} \phi} \quad \text { in } D
$$

where $K(\phi)>0$ is a coefficient of proportionality. Substituting Equation (12) into Equation (15), we obtain the following:

$$
\frac{\partial \phi}{\partial t}=-K(\phi)\left(\frac{\mathrm{d} \bar{F}\left(\chi_{\phi}\right)}{\mathrm{d} \phi}-\tau \nabla^{2} \phi\right) \quad \text { in } D .
$$

Here, we note that the derivatives $\frac{\mathrm{d} \bar{F}\left(\chi_{\phi}\right)}{\mathrm{d} \phi}$ equivalent to the topological derivatives [75-77] defined as

$$
d_{t} \bar{F}=-\frac{\partial \bar{F}\left(\chi_{\phi}\right)}{\partial \chi_{\phi}}=\lim _{\epsilon \rightarrow 0} \frac{\bar{F}\left(\Omega_{\epsilon, \mathbf{x}}\right)-\bar{F}(\Omega)}{|\xi(\epsilon)|},
$$

where $\Omega_{\epsilon, \mathbf{x}}=\Omega-\bar{B}_{\epsilon}$ is the material domain with a hole, $\bar{B}_{\epsilon}$ is a sphere of radius $\epsilon$ centered at $\mathbf{x}$ and $\xi$ is a function that decreases monotonically so that $\xi(\epsilon) \rightarrow 0$ as $\epsilon \rightarrow 0$, because the objective functional $F$ is formulated using the characteristic function $\chi_{\phi}$. As a result, in our method, topological changes that increase the number of holes are allowed, since they are equivalent to the sensitivities with respect to generating structural boundaries in the material domain. In future work, we hope to discuss the theoretical connection between the characteristic function and topological derivatives in detail. On the other hand, the level set-based structural optimization method proposed by Wang et al. [57] is essentially a type of shape optimization method, since the sensitivities have non-zero values only on the structural boundaries.

Furthermore, we assume that the boundary condition of the level set function is a Dirichlet boundary condition on the non-design boundary, and a Neumann boundary condition on the other boundaries, to represent the level set function independently of the exterior of the fixed design domain $D$. Then, the obtained time evolutionary equation with boundary conditions are summarized as follows:

$$
\left\{\begin{array}{lc}
\frac{\partial \phi}{\partial t}=-K(\phi)\left(-\frac{\partial \bar{F}\left(\chi_{\phi}\right)}{\partial \chi_{\phi}}-\tau \nabla^{2} \phi\right) & \text { in } D \\
\frac{\partial \phi}{\partial n}=0 & \text { on } \partial D \backslash \partial D_{N} \\
\phi=1 & \text { on } \partial D_{N} .
\end{array}\right.
$$

Note that Equation (18) is a reaction-diffusion equation, and that the proposed method ensures the smoothness of the level set function. 
Next, the time derivative of the regularized Lagrangian $\bar{F}_{R}$ is obtained using Equation (12) and (15) as follows:

$$
\begin{aligned}
\frac{d \bar{F}_{R}}{d t} & =\int_{D} \frac{\mathrm{d} \bar{F}_{R}}{\mathrm{~d} \phi} \frac{\partial \phi}{\partial t} d D \\
& =\int_{D} \frac{\mathrm{d} \bar{F}_{R}}{\mathrm{~d} \phi}\left(-K(\phi) \frac{\mathrm{d} \bar{F}_{R}}{\mathrm{~d} \phi}\right) d D \quad(\because(15)) \\
& =-\int_{D} K(\phi)\left(\frac{\mathrm{d} \bar{F}_{R}}{\mathrm{~d} \phi}\right)^{2} d D \quad \leq 0 .
\end{aligned}
$$

The above equation implies that when the level set function is updated based on Equations (16), the sum of the original Lagrangian $\bar{F}$ and the fictitious interface energy decreases monotonically.

\subsection{The minimum mean compliance problem}

The above proposed method is now applied to a minimum mean compliance problem. Consider a material domain $\Omega$ where the displacement is fixed at boundary $\Gamma_{u}$ and traction $\mathbf{t}$ is imposed at boundary $\Gamma_{t}$. A body force $\mathbf{b}$ may also be applied throughout the material domain $\Omega$. Let the displacement field be denoted as $\mathbf{u}$ in the static equilibrium state. The minimum compliance problem is then formulated as follows:

$$
\begin{gathered}
\qquad \inf _{\phi} F_{1}(\chi)=l(\mathbf{u}) \\
\text { subject to } \quad a(\mathbf{u}, \mathbf{v})=l(\mathbf{v}) \\
\text { for } \forall \mathbf{v} \in U \quad \mathbf{u} \in U \\
G(\chi) \leq 0
\end{gathered}
$$

where the notations in the above equation are defined as

$$
\begin{aligned}
& a(\mathbf{u}, \mathbf{v})=\int_{D} \boldsymbol{\epsilon}(\mathbf{u}): \mathbf{E}: \boldsymbol{\epsilon}(\mathbf{v}) \chi_{\phi} \mathrm{d} \Omega \\
& l(\mathbf{v})=\int_{\Gamma_{t}} \mathbf{t} \cdot \mathbf{v} \mathrm{d} \Gamma+\int_{D} \mathbf{b} \cdot \mathbf{v} \chi_{\phi} \mathrm{d} \Omega \\
& G(\chi)=\int_{D} \chi \mathrm{d} \Omega-V_{\max },
\end{aligned}
$$

where $\boldsymbol{\epsilon}$ is the linearized strain tensor, $\mathbf{E}$ is the elasticity tensor, and

$$
U=\left\{\mathbf{v}=v_{i} \mathbf{e}_{i}: v_{i} \in H^{1}(D) \text { with } \mathbf{v}=0 \text { on } \Gamma_{u}\right\}
$$


Next, the sensitivity of Lagrangian $\bar{F}_{1}$ for the minimum compliance problem is derived. The Lagrangian $\bar{F}_{1}$ is the following:

$$
\bar{F}_{1}=l(\mathbf{u})-a(\mathbf{u}, \mathbf{v})+l(\mathbf{v})+\lambda G .
$$

The sensitivity can be simply obtained using the adjoint variable method by

$$
\begin{aligned}
\left\langle\frac{\partial \bar{F}_{1}}{\partial \chi_{\phi}}, \tilde{\chi_{\phi}}\right\rangle & =\left\langle\frac{\partial l(\mathbf{u})}{\partial \mathbf{u}}, \delta \mathbf{u}\right\rangle\left\langle\frac{\partial \mathbf{u}}{\partial \chi_{\phi}}, \tilde{\chi_{\phi}}\right\rangle-\left\langle\frac{\partial a(\mathbf{u}, \mathbf{v})}{\partial \mathbf{u}}, \delta \mathbf{u}\right\rangle\left\langle\frac{\partial \mathbf{u}}{\partial \chi_{\phi}}, \tilde{\chi_{\phi}}\right\rangle \\
& -\left\langle\frac{\partial a(\mathbf{u}, \mathbf{v})}{\partial \chi_{\phi}}, \tilde{\chi_{\phi}}\right\rangle+\lambda\left\langle\frac{\partial G}{\partial \chi_{\phi}}, \tilde{\chi_{\phi}}\right\rangle
\end{aligned}
$$

where the adjoint field is defined as follows:

$$
a(\mathbf{v}, \mathbf{u})=l(\mathbf{u}) \quad \text { for } \forall \mathbf{u} \in U \quad \mathbf{v} \in U
$$

Therefore, the time evolutionary equation (18) of the minimum mean compliance problem is as follows:

$$
\frac{\partial \phi}{\partial t}=-K(\phi)\left(\boldsymbol{\epsilon}(\mathbf{u}): \mathbf{E} \chi_{\phi}: \boldsymbol{\epsilon}(\mathbf{v})-\lambda-\tau \nabla^{2} \phi\right) \quad \text { in } D
$$

\subsection{The optimum design problem of compliant mechanisms}

Next, the proposed method is applied to an optimum design problem of compliant mechanisms. Consider a material domain $\Omega$ where the displacement is fixed at boundary $\Gamma_{u}$ and traction $\mathbf{t}_{i n}$ is imposed at boundary $\Gamma_{i n}$.

Let the displacement field be denoted as $\mathbf{u}_{1}$ in the static equilibrium state. The optimum design problem of compliant mechanisms is then formulated as follows [20]:

$$
\begin{array}{cl}
\inf _{\phi} & F_{2}(\chi)=-l_{2}\left(\mathbf{u}_{1}\right) \\
\text { subject to } & a\left(\mathbf{u}_{1}, \mathbf{v}\right)=l_{1}(\mathbf{v}) \\
& \text { for } \forall \mathbf{v} \in U \quad \mathbf{u}_{1} \in U \\
& G(\chi) \leq 0,
\end{array}
$$

where the notations in the above equation are defined as

$$
\begin{aligned}
& l_{1}(\mathbf{v})=\int_{\Gamma_{\text {in }}} \mathbf{t}_{\text {in }} \cdot \mathbf{v} \mathrm{d} \Gamma \\
& l_{2}(\mathbf{v})=\int_{\Gamma_{\text {out }}} \mathbf{t}_{\text {out }} \cdot \mathbf{v} \mathrm{d} \Gamma
\end{aligned}
$$


where $\mathbf{t}_{\text {out }}$ is a dummy traction vector representing the direction of the specified deformation at output port $\Gamma_{\text {out }}$. Based on Sigmund's formulation, a nonstructural distributed spring is located at boundary $\Gamma_{\text {out }}$, and sufficient stiffness at boundary $\Gamma_{\text {out }}$ is obtained by maximizing the mutual mean compliance, since this provides a reaction force from the spring due to the deformation at boundary $\Gamma_{\text {out }}$, which serves to automatically maximize the stiffness.

Next, the sensitivity of Lagrangian $\bar{F}_{2}$ for the design of compliant mechanisms is derived. The Lagrangian $\bar{F}_{2}$ is the following:

$$
\bar{F}_{2}=-l_{2}\left(\mathbf{u}_{1}\right)+a\left(\mathbf{u}_{1}, \mathbf{v}\right)-l_{1}(\mathbf{v})+\lambda G \text {. }
$$

The sensitivity can be simply obtained using the adjoint variable method by

$$
\begin{aligned}
\left\langle\frac{\partial \bar{F}_{2}}{\partial \chi_{\phi}}, \tilde{\chi_{\phi}}\right\rangle & =-\left\langle\frac{\partial l_{2}\left(\mathbf{u}_{1}\right)}{\partial \mathbf{u}_{1}}, \delta \mathbf{u}_{1}\right\rangle\left\langle\frac{\partial \mathbf{u}_{1}}{\partial \chi_{\phi}}, \tilde{\chi_{\phi}}\right\rangle+\left\langle\frac{\partial a\left(\mathbf{u}_{1}, \mathbf{v}\right)}{\partial \mathbf{u}_{1}}, \delta \mathbf{u}_{1}\right\rangle\left\langle\frac{\partial \mathbf{u}_{1}}{\partial \chi_{\phi}}, \tilde{\chi_{\phi}}\right\rangle \\
& +\left\langle\frac{\partial a\left(\mathbf{u}_{1}, \mathbf{v}\right)}{\partial \chi_{\phi}}, \tilde{\chi_{\phi}}\right\rangle+\lambda\left\langle\frac{\partial G}{\partial \chi_{\phi}}, \tilde{\chi_{\phi}}\right\rangle
\end{aligned}
$$

where the adjoint field is defined as follows:

$$
a\left(\mathbf{v}, \mathbf{u}_{1}\right)=l_{2}\left(\mathbf{u}_{1}\right) \quad \text { for } \forall \mathbf{u}_{1} \in U \quad \mathbf{v} \in U \text {. }
$$

Therefore, the time evolutionary equation (18) of the optimum design problem of compliant mechanisms is as follows:

$$
\frac{\partial \phi}{\partial t}=-K(\phi)\left(-\boldsymbol{\epsilon}\left(\mathbf{u}_{1}\right): \mathbf{E} \chi_{\phi}: \boldsymbol{\epsilon}(\mathbf{v})-\lambda-\tau \nabla^{2} \phi\right) \quad \text { in } D
$$

\subsection{The lowest eigenfrequency maximization problem}

Next, the proposed method is applied to a lowest eigenfrequency maximization problem. Consider a fixed design domain $D$ with fixed boundary at $\Gamma_{u}$. The material domain $\Omega$ is filled with a linearly elastic material. The objective functional for the lowest eigenfrequency maximization problem can be formulated as follows:

$$
\inf _{\phi} \quad F_{3}=-\left(\sum_{k=1}^{q} \frac{1}{\omega_{k}^{2}}\right)^{-1}=-\left(\sum_{k=1}^{q} \frac{1}{\lambda_{k}}\right)^{-1},
$$

where $\omega_{k}$ is the $k$-th eigenfrequency, $\lambda_{k}$ is $k$-th eigenvalue and $q$ is an appropriate number of eigenfrequencies from the lowest eigen-mode. Therefore, the topology optimization problem, including the volume constraint, is formulated 
as follows:

$$
\begin{aligned}
& \inf _{\phi} \quad F_{3}=-\left(\sum_{k=1}^{q} \frac{1}{\lambda_{k}}\right)^{-1} \\
& \text { subject to } \quad G \leq 0 \\
& \quad a\left(\mathbf{u}_{k}, \mathbf{v}\right)=\lambda_{k} b\left(\mathbf{u}_{k}, \mathbf{v}\right) \\
& \text { for } \quad \forall \mathbf{v} \in U, \quad \mathbf{u}_{k} \in U, \quad k=1, \ldots, q,
\end{aligned}
$$

where the above notation $b\left(\mathbf{u}_{k}, \mathbf{v}\right)$ is defined in the following equation,

$$
b\left(\mathbf{u}_{k}, \mathbf{v}\right)=\int_{\Omega} \rho \mathbf{u}_{k} \cdot \mathbf{v d} \Omega
$$

where $\mathbf{u}_{k}$ is the corresponding $k$-th eigenmode and $\rho$ is the density.

Next, the sensitivity of Lagrangian $\bar{F}_{3}$ for the design of compliant mechanisms is derived. The Lagrangian $\bar{F}_{3}$ is the following:

$$
\begin{array}{r}
\bar{F}_{3}=-\left(\sum_{k=1}^{q} \frac{1}{\lambda_{k}}\right)^{-1}+\sum_{k=1}^{q} \alpha_{k}\left(a\left(\mathbf{u}_{k}, \mathbf{v}_{k}\right)-\lambda_{k} b\left(\mathbf{u}_{k}, \mathbf{v}_{k}\right)\right)+\lambda G \\
\text { for } \quad \alpha_{k} \in R
\end{array}
$$

The sensitivity can be simply obtained using the adjoint variable method by

$$
\begin{aligned}
\left\langle\frac{\mathrm{d} \bar{F}_{3}}{\mathrm{~d} \chi_{\phi}}, \tilde{\chi_{\phi}}\right\rangle & =\left(\sum_{k=1}^{q} \frac{1}{\lambda_{k}}\right)^{-2}\left[-\sum_{k=1}^{q} \frac{1}{\lambda_{k}^{2}}\left(\left\langle\frac{\partial a\left(\mathbf{u}_{k}, \mathbf{v}_{k}\right)}{\partial \chi_{\phi}}, \tilde{\chi_{\phi}}\right\rangle-\lambda_{k}\left\langle\frac{\partial b\left(\mathbf{u}_{k}, \mathbf{v}_{k}\right)}{\partial \chi_{\phi}}, \tilde{\chi_{\phi}}\right\rangle\right)\right] \\
& +\lambda\left\langle\frac{\partial G}{\partial \chi_{\phi}}, \tilde{\chi_{\phi}}\right\rangle
\end{aligned}
$$

where the adjoint field is defined as follows:

$$
a\left(\mathbf{u}_{k}, \mathbf{v}_{k}\right)=\lambda_{k} b\left(\mathbf{u}_{k}, \mathbf{v}_{k}\right) \quad \text { for } \forall \mathbf{u}_{k} \in U \quad \mathbf{v}_{k} \in U \text {. }
$$

Therefore, the time evolutionary equation (16) of the lowest eigenfrequency maximization problem is as follows:

$$
\begin{aligned}
& \frac{\partial \phi}{\partial t}=-K(\phi)\left\{\left(\sum_{k=1}^{q} \frac{1}{\lambda_{k}}\right)^{-2} \sum_{k=1}^{q}\left(\frac{\boldsymbol{\epsilon}\left(\mathbf{u}_{k}\right): \mathbf{E} \chi_{\phi}: \boldsymbol{\epsilon}\left(\mathbf{v}_{k}\right)-\lambda_{k} \rho \chi_{\phi} \mathbf{u}_{k} \cdot \mathbf{v}_{k}}{\lambda_{k}^{2}}\right)\right. \\
&\left.-\lambda-\tau \nabla^{2} \phi\right\} \text { in } D
\end{aligned}
$$




\section{Numerical implementations}

\subsection{Optimization algorithms}

The flowchart of the optimization procedure is shown in Fig. 1.

[Fig. 1 about here.]

As this figure shows, the initial configuration is first set. In the second step, the equilibrium equations are solved using the Finite Element Method. In the third step, the objective functional is computed. Here, the optimization process is finished if the objective functional has converged, otherwise the sensitivities with respect to the objective functional are computed. In the fourth step, the level set function $\phi$ is updated based on Eq.(18) using the Finite Element Method. Here, the Lagrange multiplier $\lambda$ is estimated to satisfy the following:

$$
G(\phi(t+\Delta t))=0 .
$$

In addition, the volume constraint is handled using the augmented Lagrangian method [86-88].

\subsection{Scheme of the system of time evolutionary equations}

In this research, we develop a scheme for a system of time-evolutionary equations (18). First, we introduce a characteristic length $L$ and an extended parameter $C$ to normalize the sensitivities, and Equations (18) can then be replaced by dimensionless equations as follows.

$$
\begin{cases}\frac{\partial \phi}{\partial t}=-K(\phi)\left(-C \frac{\partial \bar{F}}{\partial \chi_{\phi}}-\tau L^{2} \nabla^{2} \phi\right) & \text { in } D \\ \frac{\partial \phi}{\partial n}=0 & \text { on } \partial D \backslash \partial D_{N} \\ \phi=1 & \text { on } \partial D_{N},\end{cases}
$$

where $C$ is defined as

$$
C=\frac{c \int_{D} \mathrm{~d} \Omega}{\int_{D}\left|\frac{\partial \bar{F}}{\partial \chi_{\phi}}\right| \mathrm{d} \Omega}
$$


Next, Equations (51) are discretized in the time direction using the Finite Difference Method as follows:

$$
\left\{\begin{array}{l}
\frac{\phi(t+\Delta t)}{\Delta t}-K(\phi(t)) \tau L^{2} \nabla^{2} \phi(t+\Delta t) \\
=K(\phi(t)) C \frac{\partial \bar{F}}{\partial \chi_{\phi}}+\frac{\phi(t)}{\Delta t} \\
\phi=1 \quad \text { on } \partial D_{N} \\
\frac{\partial \phi}{\partial n}=0 \quad \text { on } \partial D / \partial D_{N},
\end{array}\right.
$$

where $\Delta t$ is the time increment. Next, the above equations are translated to a weak form as follows, so they can be discretized using the Finite Element Method.

$$
\left\{\begin{array}{c}
\int_{D} \frac{\phi(t+\Delta t)}{\Delta t} \tilde{\phi} d D+\int_{D} \nabla^{T} \phi(t+\Delta t)\left(\tau L^{2} K(\phi(t)) \nabla \tilde{\phi}\right) d D \\
=\int_{D}\left(K(\phi(t)) C \frac{\partial \bar{F}}{\partial \chi_{\phi}}+\frac{\phi(t)}{\Delta t}\right) \tilde{\phi} d D \\
\text { for } \forall \tilde{\phi} \in \tilde{\Phi} \\
\phi=1 \quad \text { on } \partial D_{N},
\end{array}\right.
$$

where $\tilde{\Phi}$ is the functional space of the level set function defined by

$$
\tilde{\Phi}=\left\{\phi(\mathbf{x}) \mid \phi(\mathbf{x}) \in H^{1}(D) \text { with } \phi=1 \text { on } \partial D_{N}\right\} .
$$

Discretizing Equation (54) using the Finite Element Method, the following equation is derived:

$$
\left\{\begin{array}{l}
\mathbf{T} \Phi(t+\Delta t)=\mathbf{Y} \\
\phi=1 \quad \text { on } \partial D_{N},
\end{array}\right.
$$

where $\Phi(t)$ is the nodal value vector of the level set function at time $t$ and $\mathbf{T}$ and $\mathbf{Y}$ are described as follows:

$$
\begin{aligned}
& \mathbf{T}=\bigcup_{j=i}^{e} \int_{V_{e}}\left(\frac{1}{\Delta t} \mathbf{N}^{T} \mathbf{N}+\nabla^{T} \mathbf{N} K(\phi(t)) \tau L^{2} \nabla \mathbf{N}\right) d V_{e} \\
& \mathbf{Y}=\bigcup_{j=i}^{e} \int_{V_{e}}\left(K(\phi(t)) C \frac{\partial \bar{F}}{\partial \chi_{\phi}}+\frac{\phi(\mathbf{x}, t)}{\Delta t}\right) \mathbf{N} d V_{e},
\end{aligned}
$$

where $e$ is the number of elements and $\bigcup_{j=i}^{e}$ represents the union set of the elements, $j$ is the number of elements and $\mathbf{N}$ is the interpolation function of the level set function.

The upper and lower limit constraints of the level set function are not satisfied when the level set function is updated based on Eq. (56). To satisfy the constraints, the level set function is replaced based on the following rule after updating the level set function.

$$
\text { if }\|\phi\|>1 \text { then } \phi=\operatorname{sign}(\phi)
$$




\subsection{Approximated equilibrium equation}

In this research the ersatz material approach is used [61]. That is, the equilibrium Equation (60) is approximated by Equation (61).

$$
\begin{aligned}
& \int_{D} \boldsymbol{\epsilon}(\mathbf{u}): \mathbf{E}: \boldsymbol{\epsilon}(\mathbf{v}) \chi \mathrm{d} \Omega=\int_{\Gamma_{t}} \mathbf{t} \cdot \mathbf{v} \mathrm{d} \Gamma+\int_{D} \mathbf{b} \cdot \mathbf{v} \chi \mathrm{d} \Omega \\
& \int_{D} \boldsymbol{\epsilon}(\mathbf{u}): \mathbf{E}: \boldsymbol{\epsilon}(\mathbf{v}) H_{a}(\phi) \mathrm{d} \Omega=\int_{\Gamma_{t}} \mathbf{t} \cdot \mathbf{v} \mathrm{d} \Gamma+\int_{D} \mathbf{b} \cdot \mathbf{v} H_{a}(\phi) \mathrm{d} \Omega,
\end{aligned}
$$

where $H_{a}(\phi)$ is the Heaviside function approximated as

$$
H_{a 1}(\phi)= \begin{cases}d & (\phi<0) \\ 1 & (0 \leq \phi)\end{cases}
$$

or

$$
H_{a 2}(\phi)= \begin{cases}d & (\phi<-w) \\ \left(\frac{1}{2}+\frac{\phi}{w}\left(\frac{15}{16}-\frac{\phi^{2}}{w^{2}}\left(\frac{5}{8}-\frac{3}{16} \frac{\phi^{2}}{w^{2}}\right)\right)\right)(1-d)+d & (-w<\phi<w) \\ 1 & (w<\phi),\end{cases}
$$

where $w$ represents the width of transition and $d>0$ represents the ratio of material constants, namely, the Young's modulus values between the void and material domains. Parameter $d$ is introduced to ensure stable analyses of the fixed design domain when using the Finite Element method. In this research, the volume constraint function $G(\Omega)$ which is defined by Equation (9) is also approximated, as follows:

$$
G(\phi)=\int_{D} H_{g}(\phi) \mathrm{d} \Omega-V_{\max } .
$$

As shown in the following equation, $H_{g}(\phi)$ is the smoothed Heaviside function whose width of transition is 2, since as shown in Equation (7), the level set function values range from -1 to 1 .

$$
H_{g}(\phi)= \begin{cases}0 & (\phi=-1) \\ \frac{1}{2}+\frac{\phi}{2}\left(\frac{15}{16}-\frac{\phi^{2}}{4}\left(\frac{5}{8}-\frac{3}{64} \phi^{2}\right)\right) & (-1<\phi<1) \\ 1 & (\phi=1)\end{cases}
$$

We note that intermediate regions between the material and void domains are not allowed in the approximation with respect to the material distribution (61), which eliminates grayscales completely. In the approximation with respect to the volume calculation (64), intermediate regions are allowed for numerical stability. Elimination of grayscales is important when using the equilibrium equations but is not important in the volume calculation. 


\section{Numerical examples}

\subsection{Two-dimensional minimum mean compliance problems}

In this subsection, several numerical examples are presented to confirm the utility and validity of proposed optimization method for two and three dimensional minimum compliance problems. In these examples, the isotropic linear elastic material has Young's modulus $=210 \mathrm{GPa}$, Poisson's ratio $=0.31$ and parameter $d$ in approximated Heaviside function (62) is set to $1 \times 10^{-3}$. Figure 2 shows the fixed design domain and the boundary conditions of model $\mathrm{A}$ and Figure 3 shows the same for model B.

[Fig. 2 about here.]

[Fig. 3 about here.]

\subsubsection{Effect of the initial configurations}

First, using model A, we examine the effect of different initial configurations upon the resulting optimal configurations. The regularization parameter $\tau$ is set to $1 \times 10^{-4}$, parameter $c$ is set to 0.5 and the characteristic length $L$ is set to $1 \mathrm{~m}$. Parameter $K(\phi)$ is set to 1 , the upper limit of the volume constraint $V_{\max }$ is set to $40 \%$ of the volume of the fixed design domain and parameter $d$ in approximated Heaviside function (62) is set to $1 \times 10^{-3}$.

The fixed design domain is discretized using a structural mesh and four-node quadrilateral plane stress elements whose length is $6.25 \times 10^{-3} \mathrm{~m}$. Figure 4 shows four cases and their obtained optimal configurations, each using a different initial configuration. The initial configuration for Case 1 has the material domain filled with material; for Case 2, the initial configuration has two holes; for Case 3, the initial configuration has many holes; and for Case 4, the initial configuration has material filling the material domain in the upper half of the fixed design domain.

[Fig. 4 about here.]

In all cases, the optimal configurations are smooth, clear and nearly the same. That is, an appropriate optimal configuration was obtained for all initial configurations. We confirm that the dependency of the obtained optimal configurations upon the initial configurations is extremely low. 


\subsubsection{Effect of finite element mesh size}

Second, using model A, we examine the effect of the finite element mesh size upon the resulting optimal configurations. The regularization parameter $\tau$ is set to $8 \times 10^{-5}$, parameter $c$ is set to 0.2 , the characteristic length $L$ is set to $1 \mathrm{~m}$, parameter $K(\phi)$ is set to 1 , the upper limit of the volume constraint $V_{\max }$ is set to $40 \%$ of the volume of the fixed design domain and parameter $d$ in approximated Heaviside function (62) is set to $1 \times 10^{-3}$. The initial configurations in all cases have the material domain filled with material in the Fixed design domain. The fixed design domain is discretized using a structural mesh and four-node quadrilateral plane stress elements. We examine three cases whose degree of discretization is subject to the following mesh parameters: $80 \times 60$, $160 \times 120$ and $320 \times 240$. Figure 5 shows the optimal configuration for each case.

[Fig. 5 about here.]

Again, all obtained optimal configurations are smooth, clear and practically identical. That is, an appropriate optimal configuration can be obtained regardless of which degree of discretization was used here. We confirm that dependency with regard to the finite element mesh size is extremely small provided that the finite element size is sufficiently small.

\subsubsection{Effect of the regularization parameter $\tau$}

We now examine the effect that different regularization parameter $\tau$ values have upon the resulting optimal configurations. In model A, parameter $c$ is set to 0.5 , the characteristic length $L$ is set to $1 \mathrm{~m}$, parameter $K(\phi)$ is set to 1 , the upper limit of the volume constraint $V_{\max }$ is set to $40 \%$ of the volume of the fixed design domain and parameter $d$ in approximated Heaviside function (62) is set to $1 \times 10^{-3}$. The initial configuration in all case has the material domain filled with material in the fixed design domain. The fixed design domain is discretized using a structural mesh and four-node quadrilateral plane stress elements whose length is $6.25 \times 10^{-3} \mathrm{~m}$. We examine four cases where the regularization parameter $\tau$ is set to $5 \times 10^{-4}, 5 \times 10^{-5}, 3 \times 10^{-5}$ and $2 \times 10^{-5}$, respectively. Figure 6 shows the optimal configuration for each case.

[Fig. 6 about here.]

Next, using model B, parameter $c$ is set to 0.5 , the characteristic length $L$ is set to $1 \mathrm{~m}$, and the upper limit of the volume constraint $V_{\max }$ is set to $50 \%$ of the volume of the fixed design domain. The initial configurations again have the material domain filled with material in the fixed design domain. The fixed design domain is discretized using a structural mesh and four-node quadrilateral plane stress elements whose length is $6.25 \times 10^{-3} \mathrm{~m}$. We examine 
four cases where the regularization parameter $\tau$ is set to $5 \times 10^{-4}, 2 \times 10^{-4}$, $1 \times 10^{-4}$ and $1 \times 10^{-5}$, respectively. Figure 7 shows the optimal configuration for each case.

[Fig. 7 about here.]

The obtained optimal configurations are smooth and clear and we can confirm that the use of the proposed method's $\tau$ parameter allows the complexity of the optimal structures to be adjusted at will.

\subsubsection{Effect of the proportional coefficient $K(\phi)$}

Next, we now examine the effect that different definitions of proportionality coefficient $K(\phi)$ have upon the resulting optimal configurations, using four initial configurations. The fixed design domain and boundary condition are shown in Figure 8. The isotropic linear elastic material has Young's modulus $=210 \mathrm{GPa}$, Poisson's ratio $=0.31$ and parameter $d$ and $w$ in approximated Heaviside function (63) is set to $1 \times 10^{-3}$ and 1 , respectively. Parameter $c$ is set to 0.5 , the characteristic length $L$ is set to $1 \mathrm{~m}$, regularization parameter $\tau$ is set to $5 \times 10^{-4}$ and the upper limit of the volume constraint $V_{\max }$ is set to $40 \%$ of the volume of the fixed design domain. The fixed design domain is discretized using a structural mesh and four-node quadrilateral plane stress elements.

[Fig. 8 about here.]

We examine three cases, where the coefficient of proportionality $K(\phi)$ is set as follows:

$$
\begin{aligned}
& K_{\cos }(\phi)=\frac{1}{2}+\cos \left(\frac{\pi}{2} \phi\right) \\
& K_{\sin }(\phi)=1+\frac{1}{2} \sin \left(\frac{\pi}{2} \phi\right) \\
& K_{1}(\phi)=1
\end{aligned}
$$

Figure 9 shows the different initial and optimal configurations for each case.

[Fig. 9 about here.]

In all cases, the optimal configurations are smooth, clear and nearly the same. That is, an appropriate optimal configuration was obtained for all three definitions of $K(\phi)$, and we confirm that the dependency of the obtained optimal configurations upon these definitions is extremely low. 


\subsection{Three-dimensional minimum mean compliance problems}

\subsubsection{Effect of the regularization parameter $\tau$}

First, we now examine the effect that different values of the regularization parameter $\tau$ have upon the resulting optimal configurations in a three dimensional design problem. The isotropic linearly elastic material has Young's modulus $=210 \mathrm{GPa}$ and Poisson's ratio $=0.31$. Figure 10 shows the fixed design domain and boundary conditions.

[Fig. 10 about here.]

Parameter $c$ is set to 0.5 , the characteristic length $L$ is set to $1 \mathrm{~m}$, and the upper limit of the volume constraint $V_{\max }$ is set to $40 \%$ of the volume of the fixed design domain. The initial configurations have the material domain filled with material in the fixed design domain. The fixed design domain is discretized using a structural mesh and eight-node hexahedral elements whose length is $1 \times 10^{-2} \mathrm{~m}$. We examine two cases where the regularization parameter $\tau$ is set to $2 \times 10^{-4}$ and $2 \times 10^{-5}$, respectively. Figure 11 shows the optimal configuration for each case.

[Fig. 11 about here.]

The obtained optimal configurations are smooth and clear, and we can confirm that the use of the proposed method's $\tau$ parameter allows the complexity of the optimal structures to be adjusted at will for the three-dimensional case as well.

\subsubsection{Discretization using a nonstructural mesh}

Second, we show a design problem of a mechanical part model where a nonstructural mesh is employed. The isotropic linear elastic material has Young's modulus $=210 \mathrm{GPa}$ and Poisson's ratio $=0.31$. The regularization parameter $\tau$ is set to $5 \times 10^{-5}$, parameter $c$ is set to 0.5 , the characteristic length $L$ is set to $1 \mathrm{~m}$, and the upper limit of the volume constraint $V_{\max }$ is set to $45 \%$ of the volume of the design domain. The initial configurations have the material domain filled with material in the fixed design domain. Figure 12 shows the fixed design domain, boundary conditions and obtained optimal configuration.

[Fig. 12 about here.]

As shown, the obtained optimal configuration obtained by the proposed method is smooth and clear when a unstructublue mesh is used. 


\subsubsection{Uniform cross-section surface constraint}

Next, we consider the use of a uniform cross-section surface constraint, which is important from a manufacturing standpoint. A geometrical constraint can easily be imposed by using an anisotropic variation of the regularization parameter $\tau$. That is, if a component in the constraint direction of regularization parameter $\tau$ is set to a large value, the level set function will be constant in the constraint direction. As a result, in this scenario, obtained optimal configurations will reflect the imposition of a uniform cross-section surface constraint. Here, we show the effect that a uniform cross-section surface constraint has upon the obtained optimal configuration for a three-dimensional case. The isotropic linear elastic material has Young's modulus $=210 \mathrm{GPa}$ and Poisson's ratio $=0.31$. Figure 13 shows the fixed design domain and boundary conditions.

[Fig. 13 about here.]

Parameter $c$ is set to 0.5 , the characteristic length $L$ is set to $1 \mathrm{~m}$, and the upper limit of the volume constraint $V_{\max }$ is set to $30 \%$ of the volume of the design domain. The initial configurations have the material domain filled with material in the fixed design domain. The fixed design domain is discretized using a structural mesh and eight-node hexahedral elements whose length is $1 \times 10^{-2} \mathrm{~m}$. Case (a) has an isotropic regularization parameter $\tau=4 \times 10^{-5}$ as a non-uniform cross-section surface case. Case (b) has anisotropic component coefficients of the regularization parameter applied, where $\tau=4 \times 10^{-5}$ in direction $\mathbf{x}_{1}$ and $\mathbf{x}_{2}$, and $\tau=4$ in direction $\mathbf{x}_{3}$, so that a uniform crosssection constraint is imposed in direction $\mathbf{x}_{3}$. Figure 14 shows the optimal configuration for the two cases.

[Fig. 14 about here.]

The obtained optimal configurations are smooth and clear, and we can confirm that our method can successfully impose a uniform cross-section surface constraint.

\subsection{Optimum design problem for a compliant mechanism}

\subsubsection{Two-dimensional compliant mechanism design problem}

Next, our proposed method is applied to the problem of finding an optimum design for a compliant mechanism. The isotropic linear elastic material has Young's modulus $=210 \mathrm{GPa}$ and Poisson's ratio $=0.31$. Figure 15 shows the fixed design domain and boundary conditions. 
[Fig. 15 about here.]

Parameter $c$ is set to 0.5 , characteristic length $L$ is set to $100 \mu \mathrm{m}$, regularization parameter $\tau$ is set to $1 \times 10^{-4}$ and the upper limit of the volume constraint $V_{\max }$ is set to $25 \%$ of the volume of the fixed design domain. The approximated Heaviside function (63) is used. Parameter $d$ is set to $1 \times 10^{-3}$ and $w$ is set to 1 . The initial configurations have the material domain filled with material in the fixed design domain. The fixed design domain is discretized using a structural mesh and four-node quadrilateral elements whose length is $0.5 \mu \mathrm{m}$. Figure 16 shows the optimal configuration and the deformed shape.

[Fig. 16 about here.]

As shown, the obtained optimal configuration is smooth and clear, and we can confirm that the obtained optimal configuration deforms in the specified direction.

\subsubsection{Three-dimensional compliant mechanism design problem}

We applied the proposed method to a three-dimensional compliant mechanism design problem and consider the use of a uniform cross-section surface constraint. The isotropic linear elastic material has Young's modulus $=210$ GPa and Poisson's ratio $=0.31$. Figure 17 shows the fixed design domain and boundary conditions.

[Fig. 17 about here.]

Parameter $c$ is set to 0.5 , characteristic length $L$ is set to $100 \mu \mathrm{m}$ and the upper limit of the volume constraint $V_{\max }$ is set to $20 \%$ of the volume of the fixed design domain. The approximated Heaviside function (63) is used, parameter $d$ is set to $1 \times 10^{-3}$ and $w$ is set to 1 . The initial configurations have the material domain filled with material in the fixed design domain. The fixed design domain is discretized using a structural mesh and eightnode hexahedral elements whose length is $1 \mu \mathrm{m}$. Case (a) has an isotropic regularization parameter $\tau=1 \times 10^{-4}$ as a non-uniform cross-section surface case. Case (b) has anisotropic component coefficients of the regularization parameter applied, where $\tau=1 \times 10^{-4}$ in directions $\mathbf{x}_{1}$ and $\mathbf{x}_{3}$, and $\tau=$ $5 \times 10^{-1}$ in direction $\mathbf{x}_{2}$, so that a uniform cross-section constraint is imposed in direction $\mathbf{x}_{2}$. Figure 18 shows the optimal configurations.

[Fig. 18 about here.]

As shown, the obtained optimal configurations are smooth and clear, and we can confirm that our method can successfully impose a uniform cross-section surface constraint. 


\subsection{The lowest eigenfrequency maximization problem}

\subsubsection{Two-dimensional design problem}

Finally, the proposed method is applied to the lowest eigenfrequency maximization problem. The isotropic linear elastic material has Young's modulus $=210 \mathrm{GPa}$, Poisson's ratio $=0.31$ and mass density $=7,850 \mathrm{~kg} / \mathrm{m}^{3}$. Figure 19 shows the fixed design domain and boundary conditions for the twodimensional lowest eigenfrequency maximization problem.

[Fig. 19 about here.]

As shown, the right and left sides of the fixed design domain are fixed and a concentrated mass $\mathrm{M}=1 \mathrm{~kg}$ is set at the center of the fixed design domain. The fixed design domain is discretized using a structural mesh and four-node quadrilateral elements whose length is $5 \times 10^{-3} \mathrm{~m}$. Parameter $c$ is set to 0.5 , characteristic length $L$ is set to $1 \mathrm{~m}, K(\phi)$ is set to 1 and the upper limit of the volume constraint $V_{\max }$ is set to $50 \%$ of the volume of the fixed design domain. The Approximated Heaviside function (62) is used, and parameter $d$ is set to $1 \times 10^{-2}$. We examine three cases where parameter $\tau$ is set to $1.0 \times 10^{-4}$, $1.0 \times 10^{-5}$, and $1.0 \times 10^{-6}$, respectively. Figure 20 shows the obtained optimal configurations .

[Fig. 20 about here.]

The obtained optimal configurations are smooth and clear, and we can confirm that the use of the proposed method's $\tau$ parameter allows the complexity of the optimal structures to be adjusted at will for the lowest eigenfrequency maximization problem as well.

\subsubsection{Three-dimensional design problem}

Figure 21 shows the fixed design domain and boundary conditions for a threedimensional lowest eigenfrequency maximization problem.

[Fig. 21 about here.]

The isotropic linear elastic material has Young's modulus $=210$ GPa, Poisson's ratio $=0.31$, mass density $=7,850 \mathrm{~kg} / \mathrm{m}^{3}$ and a concentrated mass $M=80 \mathrm{~kg}$ is set at the center of the fixed design domain. The fixed design domain is discretized using a structural mesh and eight-node hexahedral elements whose length is $1 \times 10^{-3} \mathrm{~m}$. Parameter $c$ is set to 0.5 , characteristic length $L$ is set to $1 \mathrm{~m}, K(\phi)$ is set to 1 and the upper limit of the volume constraint $V_{\max }$ is set to $30 \%$ of the volume of the fixed design domain. The Ap- 
proximated Heaviside function (62) is used, and parameter $d$ is set to $1 \times 10^{-2}$. Figure 22 shows the optimal configurations.

[Fig. 22 about here.]

As shown, the obtained optimal configurations are smooth and clear.

\section{Conclusions}

This paper proposed a new topology optimization method incorporating level set boundary expressions based on the concept of the phase field method and applied it to minimum mean compliance problems, optimum compliant mechanism design problems, and lowest eigenfrequency maximization problems. We achieved the following:

(1) A topology optimization method was formulated, incorporating level set boundary expressions, where the optimization problem is handled as a problem to minimize the energy functional including a fictitious interface energy. Furthermore, a method for solving the optimization problem using a reactiondiffusion equation was proposed.

(2) Based on the proposed topology optimization method, minimum mean compliance problems, optimum design problem of compliant mechanisms, and lowest eigenfrequency maximization problems were formulated, and an optimization algorithm was then constructed. A scheme for updating the level set function using a time evolutional equation was proposed.

(3) Several numerical examples were provided to confirm the usefulness of the proposed topology optimization method for the various problems examined in this paper. We confirmed that smooth and clear optimal configurations were obtained using the proposed topology optimization method, which also allows control of the geometrical complexity of the obtained optimal configurations. The obtained optimal configurations show minimal dependency upon the finite element size or initial configurations. In addition, we showed that uniform cross-section surface constraints can easily be imposed by using an anisotropic variation of the regularization parameter $\tau$.

\section{Acknowledgments.}

The authors sincerely appreciate the support received from JSPS Scientific Research (C), No. 19560142, and JSPS Fellows. 


\section{References}

[1] J. W. Cahn and J. E. Hilliard, Free energy of a nonuniform system. I. interfacial free energy, The Journal of Chemical Physics, 28 (2) (1958) 258-267.

[2] S. M. Allen, J. W. Cahn, A microscopic theory for antiphase boundary motion and its application to antiphase domain coarsening, Acta Metall, 27 (1979) 1085-1095.

[3] D. Eyre, Systems of Cahn-Hilliard equations, SIAM Journal on Applied Mathematics, 53 (6) (1993) 1686-1712.

[4] P. H. Leo, J. S. Lowengrub, H. J. Jou, A diffuse interface model for microstructural evolution in elastically stressed solids, Acta Metall, 46 (6) (1998) 2113-2130.

[5] W. Prager, A note on discretized michell structures, Computer Methods in Applied Mechanics and Engineering, 3 (3) (1974) 349.

[6] K. Svanberg, Optimal geometry in truss design, In Foundations of Structural Optimization: A Unified Approach, Wiley, New York, 1982.

[7] O. C. Zienkiewicz, J. S. Campbell, Shape optimization and sequential linear programming, In Optimum Structural Design. Wiley, New Yourk, 1973.

[8] J. Haslinger, R. A. E. Mälinen, Introduction to shape optimization: theory, approximation and Computation, SIAM, Philadelphia, 2003.

[9] B. Mohammadi, O. Pironneau, Applied shape optimization for fluids, Oxford University Press, Oxford, 2001.

[10] O. Pironneau, Optimal shape design for elliptic systems, Springer-Verlag, New York, 1984.

[11] J. Sokolowski, J. P. Zolesio, Introduction to shape optimization: shape sensitivity analysis, Springer-Verlag, Berlin, 1992.

[12] M. P. Bendsøe, N. Kikuchi, Generating optimal topologies in structural design using a homogenization method, Computer Methods in Applied Mechanics and Engineering, 71 (1988) 197-224.

[13] M. P. Bendsøe, O. Sigmund, Topology optimization: theory, methods, and applications, Springer-Verlag, Berlin, 2003.

[14] A. Cherkaev, Variational methods for structural optimization, Springer-Verlag, New York, 2000.

[15] K. Suzuki, N. Kikuchi, A homogenization method for shape and topology optimization, Computer Methods in Applied Mechanics and Engineering, 93 (1991) 291-318. 
[16] A. R. Diaz, N. Kikuchi, Solutions to shape and topology eigenvalue optimization using a homogenization method, International Journal for Numerical Methods in Engineering, 35 (1992) 1487-1502.

[17] Z. D. Ma, N. Kikuchi, Topological design for vibrating structures, Computer Methods in Applied Mechanics and Engineering, 121 (1995) 259-280.

[18] X. Y. Yang, Y. M. Xie, G. P. Steven, O. M. Querin, Topology optimization for frequencies using an evolutionary method, Journal of Structural Engineering, 125 (1999) 1432-1438.

[19] S. Nishiwaki, M. I. Frecker, S. Min and N. Kikuchi, Topology optimization of compliant mechanisms using the homogenization method, International Journal of Numerical Methods in Engineering, 42 (1998) 535-559.

[20] O. Sigmund, On the design of compliant mechanisms using topology optimization, Mechanics Based Design of Structures and Machines, 25 (4) (1997) 493-524.

[21] J. Haslinger, A. Hillebrand, T. Karkkainen, M. Miettinen, Optimization of conducting structures by using the homogenization method, Structural and multidisciplinary optimization, 24 (2002) 125-140.

[22] Q. Li, G. P. Steven, O. M. Querin, Y. M. Xie, Shape and topology design for heat conduction by evolutionary structural optimization, International Journal of Heat and Mass Transfer, 42 (1999) 3361-3371.

[23] A. Iga, S. Nishiwaki, K. Izui, M. Yoshimura, Topology optimization for thermal conductors with heat convection and conduction including design-dependent effects, International Journal of Heat and Mass Transfer, 52 (2009) 2721-2732.

[24] F. Murat, L. Tartar, Optimality conditions and homogenization, Proceedings of Nonlinear Variational Problems, Pitman Publishing Program, Boston, (1985) $1-8$.

[25] G. Allaire, Shape optimization by the homogenization method. Springer, Verlag, 2002.

[26] A. Benssousan, J. L. Lions, G. Papanicoulau, Asymptotic analysis for periodic structures. North-Holland, Amsterdam-New York-Oxford, 1978.

[27] E. Sanchez-Palencia, Non homogeneous media and vibration theory, Lecture notes in physics 127. Springer-Verlag, Berlin, 1980.

[28] J. L. Lions, Some methods in mathematical analysis of systems and their control, Beijing, Kexue Chubanshe Science Press and Gordon \& Breach Science Pub. 1981.

[29] M. P. Bendsøe, Optimal shape design as a material distribution problem, Structural Optimization, 1 (1989) 193-202.

[30] M. P. Bendsøe, O. Sigmund, Optimization of Structural Topology, Shape and Material, Springer, Berlin, 1997. 
[31] R. J. Yang, C. H. Chung, Optimal topology design using linear programming, Computers and Structures, 53 (1994) 265-275.

[32] M. P. Bendsøe, O. Sigmund, Material interpolation schemes in topology optimization, Archive of Applied Mechanics, 69 (1999) 635-654.

[33] M. Y. Wang, S. Zhou, Phase field: a variational method for structural topology optimization, Computer Modeling in Engineering and Science, 6 (6) (2004) 547566.

[34] S. Zhou, M. Y. Wang, Multimaterial structural topology optimization with a generalized Cahn-Hilliard model of multiphase transition structural and multidisciplinary optimization, Structural and Multidisciplinary Optimization, 33 (2) (2007) 89-111.

[35] S. Zhou, M. Y. Wang, 3D Multi-material structural topology optimization with the generalized Cahn-Hilliard equations, Computer Modeling in Engineering and Science, 16 (2) (2006) 83-101.

[36] M. Y. Wang, S. Zhou, H. Ding, Nonlinear diffusions in topology optimization, Structural and Multidisciplinary Optimization, 28 (2004) 262-276.

[37] M. Y. Wang, S. Zhou, Synthesis of shape and topology of multi-material structures with a phase-field method, Journal of Computer-Aided Materials Design, 11 (2004) 117-138.

[38] M. Burger, R. Stainko, Phase-field relaxation of topology optimization with local stress constraints, SIAM Journal on Control and Optimization, 45 (4) (2006) 1447-1466.

[39] Y. M. Xie, G. P. Steven, A simple evolutionary procedure for structural optimization, Computers and Structures, 49 (1993) 885-896.

[40] A. Z. Diaz, O. Sigmund, Checkerboard patterns in layout optimization, Structural Optimization, 10 (1995) 40-45.

[41] O. Sigmund, J. Petersson, Numerical instabilities in topology optimization: a survey on procedures dealing with checkerboards, mesh-dependencies and local minima, Structural Optimization, 16 (1998) 68-75.

[42] R. B. Haber, C. S. Jog, M. P. Bendsøe, A new approach to variable-topology shape design using a constraint on perimeter, Structural and Multidisciplinary Optimization, 11 (1-2) (1996) 1-12.

[43] J. Petersson, O. Sigmund, Slope constrained topology optimization, International Journal for Numerical Methods in Engineering, 41 (8) (1998) $1417-1434$.

[44] M. Zhou, Y. K. Shyy, H. L. Thomas, Checkerboard and minimum member size control in topology optimization method, Structural and Multidisciplinary Optimization, 21 (2) (2001) 152-158. 
[45] S. Osher, J. A. Sethian, Front propagating with curvature dependent speed: algorithms based on the Hamilton-Jacobi formulations, Journal of Computational Physics, 78 (1988) 12-49.

[46] M. Sussman, P. Smereka, S. Osher, A level set approach for computing solutions to incompressible two-phase flow, Journal of Computational Physics, 114 (1994) 146-159.

[47] Y. C. Chang, T. Y. Hou, B. Merriman, S. Osher, A level set formulation of Eulerian interface capturing methods for incompressible fluid flow, Journal of Computational Physics, 124 (1996) 449-464.

[48] M. Susmman, E. G. Puckett, A coupled level set and volume-of-fluid method for computing 3D and axisymmetric incompressible two-phase flows, Journal of Computational Physics, 162 (2000) 301-337.

[49] G. Son, V. K. Dhir, Numerical simulation of film boiling near critical pressures with a level set method, Journal of Heat Transfer, 120 (1998) 183-192.

[50] J. C. Ye, Y. Bresler, P. Moulin, A self-referencing level-set method for image reconstruction from sparse fourier samples, International Journal of Computer Vision, 50 (2002) 253-270.

[51] R. Tsai, S. Osher, Level set methods and their applications in image science, Communications in Mathematical Sciences, 1 (2003) 623-656.

[52] K. Doel, U. M. Ascher, On level set regularization for highly ill-posed distributed parameter estimation problems, Journal of Computational Physics, 216 (2006) 707-723.

[53] J. A. Sethian, Level set method and fast marching methods, Cambridge Monographs on Applied and Computational Mechanics (2nd edn), Cambridge University Press, Cambridge, U. K., 1999.

[54] J. A. Sethian, A. Wiegmann, A structural boundary design via level-set and immersed interface methods, Journal of Computational Physics, 163 (2000) 489-528.

[55] S. Osher, F. Santosa, Level-set methods for optimization problems involving geometry and constraints: frequencies of a two-density inhomogeneous drum, Journal of Computational Physics, 171 (2001) 272-288.

[56] T. Belytschko, S. P. Xiao, C. Parimi, Topology optimization with implicit functions and regularization, International Journal for Numerical Methods in Engineering, 57 (2003) 1177-1196.

[57] M. Y. Wang, X. Wang, D. Guo, A level set method for structural topology optimization, Computer Methods in Applied Mechanics and Engineering, 192 (2003) 227-246.

[58] M. Y. Wang, X. Wang, "Color" level sets: a multi-phase method for structural topology optimization with multiple materials, Computer Methods in Applied Mechanics and Engineering, 193 (2004) 469-496. 
[59] J. Luo, Z. Wang, S. Chen, L. Tong, M. Y. Wang, A new level set method for systematic design of hinge free compliant mechanisms, Computer Methods in Applied Mechanics and Engineering, 198 (2004) 318-331.

[60] S. Chen, M. Y. Wang, A. Q. Liu, Shape feature control in structural topology optimization, Computer-Aided Design, 40 (2008) 951-962.

[61] G. Allaire, F. Jouve, A. M. Toader, Structural optimization using sensitivity analysis and a level-set method, Journal of Computational Physics, 194 (2004) 363-393.

[62] G. Allaire, F. Jouve, A level-set method for vibration and multiple loads structural optimization, Computer Methods in Applied Mechanics and Engineering, 194 (2005) 3269-3290.

[63] J. Chen, V. Shapiro, K., I. Tsukanov, Shape optimization with topological changes and parametric control, International Journal for Numerical Methods in Engineering, 71 (2007) 313-346.

[64] S. Y. Wang, M. Y. Wang, A moving superimposed finite element method for structural topology optimization, International Journal for Numerical Methods in Engineering, 65 (2006) 1892-1922.

[65] P. Wei, M. Y. Wang, Piecewise constant level set method for structural topology optimization, International Journal for Numerical Methods in Engineering, 78 (4) (2008) 379-402.

[66] Z. Luo, L. Tong, J. Luo, P. Wei, M. Y. Wang, Design of piezoelectric actuators using a multiphase level set method of piecewise constants, Journal of Computational Physics, 228 (2009) 2643-2659.

[67] Z. Luo, L. Tong, H. Ma, Shape and topology optimization for electrothermomechanical microactuators using level set methods, Journal of Computational Physics, 228 (2009) 3173-3181.

[68] S. Park, S. Min, Magnetic actuator design for maximizing force using level set based topology optimization, IEEE transactions on magnetics, 45 (5) (2009) 2336-2339.

[69] H. Shim, V. T. T. Ho, S. Wang, D. A. Tortorelli, Topological shape optimization of electromagnetic problems using level set method and radial basis function, Computer Moldering in Engineering and Science, 37 (2) (2008) 175-202.

[70] T. Yamada, S. Yamasaki, S. Nishiwaki, K. Izui, M. Yoshimura, Design of compliant thermal actuator using structural optimization based on the level set method, Journal of Computing and Information Science in Engineering, (submitted).

[71] J. H. Rong, Q. Q. Liang, A level set method for topology optimization of continuum structures with bounded design domains, Computer Methods in Applied Mechanics and Engineering, 197 (2008) 1447-1465. 
[72] T. Yamada, S. Yamasaki, S. Nishiwaki, K. Izui, M. Yoshimura, A study of boundary settings in the design domain for structural optimization based on the Level set method, Transactions of the Japan Society for Industrial and Applied Mathematics, 18 (3) (2008) 487-505.

[73] G. Allaire, F. Gournay, F. Jouve, A. M. Toader, Structural optimization using topological and shape sensitivity via a level set method. Internal Report 555, Ecole Polytechnique, France, (2004).

[74] H. A. Eschenauer, V. Kobelev, A. Schumacher, Bubble method for topology and shape optimization of structures, Structural Optimization, 8 (1994) 42-51.

[75] J. Sokolowski, A. Zochowski, On the topological derivatives in shape optimization, SIAM Journal on Control and Optimization, 37 (1999) 1251-1272.

[76] L. He, C. Y. Kao, S. Osher, Incorporating topological derivatives into shape derivatives based level set methods, Journal of Computational Physics, 225 (2007) 891-909.

[77] A. A. Novotny, R. A. Feijóo, E. Taroco, C. Padra, Topological sensitivity analysis, Computer Methods in Applied Mechanics and Engineering, 192 (2003) 803-829.

[78] S. Y. Wang, K. M. Lim, B. C. Khoo, M. Y. Wang, An extended level set method for shape and topology optimization, Journal of Computational Physics, 221 (2007) 395-421.

[79] D. L. Chopp, Computing minimal surfaces via level set curvature flow, Journal of Computational Physics, 106 (1993) 77-91.

[80] J. A. Sethian, Level set methods and fast marching methods, Cambridge University Press, New York, 1999.

[81] M. sussman, P. Smereka, S. Osher, A level set approach for computing solutions to incompressible two-phase flow, Journal of Computational Physics, 114 (1994) 146-159.

[82] A. N. Tikhonov, V. Y. Arsenin, Solutions of ill-posed problems, Winston and Sons, Washington, D.C., 1997.

[83] A. Braides, $\Gamma$-convergence for Beginners, Oxford Lecture Series In Mathematics and Its Applications 22, Oxford University Press, Oxford, 2002.

[84] A. Leitao, O. Scherzer, On the relation between constraint regularization, level sets and shape optimization, Inverse Problems, 19 (2003) 1-11.

[85] L. Ambrosio, G. Buttazzo, An optimal design problem with perimeter penalization, Calculus of Variations and Partial Differential Equations, 1 (1) (1993) 55-69.

[86] M. R. Hestenes, Multiplier, gradient methods, Journal of Optimization Theory and Applications, 4 (5) (1969) 303-320. 
[87] M. Powell, A method for nonlinear constraints in minimization problems, Optimization, Academic Press, London, England, (1969) 283-298.

[88] R. T. Rockafellar, The multiplier method of Hestenes and Powell applied to convex programming, Journal of Optimization Theory and Applications, 12 (1973) 555-562. 


\section{List of Figures}

$1 \quad$ Flowchart of optimization procedure

2 Fixed design domain and boundary conditions of model A

3 Fixed design domain and boundary conditions of model B

4 Initial configurations, intermediate results and optimal configurations

5 Optimal configurations: (a) $80 \times 60$ mesh; (b) $160 \times 120$ mesh;

(c) $320 \times 240$ mesh

$6 \quad$ Optimal configurations: (a) $\tau=5 \times 10^{-4}$; (b) $\tau=5 \times 10^{-5}$; (c) $\tau=3 \times 10^{-5} ;$ (d) $\tau=2 \times 10^{-5}$

$7 \quad$ Initial configurations, intermediate results and optimal configurations: (a) $\tau=5 \times 10^{-4}$; (b) $\tau=2 \times 10^{-4}$; (c) $\tau=1 \times 10^{-4} ;$ (d) $\tau=1 \times 10^{-5}$

8 Fixed design domain and boundary conditions of model $\mathrm{C}$

$9 \quad$ Initial configurations and optimal configurations

10 Fixed design domain and boundary conditions for three dimensional design problem

11 Optimal configurations: (a) $\tau=2 \times 10^{-4}$; (b) $\tau=2 \times 10^{-5}$

12 Fixed design domain, boundary conditions and optimal configuration for a mechanical part model

13 Fixed design domain and boundary conditions

14 Optimal configurations: (a) Non-uniform cross-section surface; (b) Uniform cross-section surface

15 Fixed design domain for a two-dimensional compliant mechanism

16 Configurations of the two-dimensional compliant mechanism
(a) Optimal configuration;
(b) Deformed shape

17 Fixed design domain for a three-dimensional compliant mechanism 
18 Configurations of the three-dimensional the compliant mechanisms: (a) Non-uniform cross-section surface (b) Uniform cross-section surface

19 Fixed design domain for the two-dimensional the lowest eigenfrequency maximization problem

20 Optimal configurations for the two-dimensional lowest eigenfrequency maximization problem: (a) regularization parameter $\tau=1.0 \times 10^{-4}$; (b) regularization parameter $\tau=1.0 \times 10^{-5} ;$ (c) regularization parameter $\tau=1.0 \times 10^{-6}$

21 Fixed design domain for the three-dimensional lowest eigenfrequency maximization problem

22 Optimal configurations of the three-dimensional lowest eigenfrequency maximization problem 


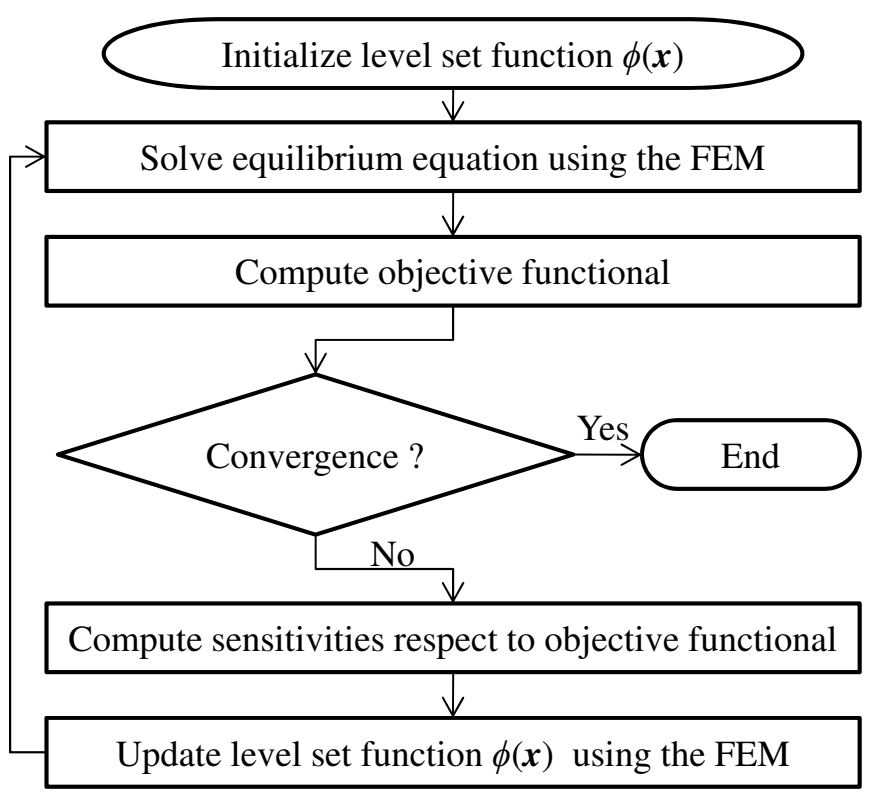

Fig. 1. Flowchart of optimization procedure 


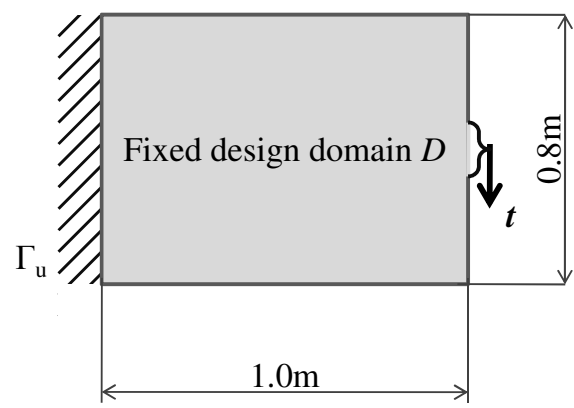

Fig. 2. Fixed design domain and boundary conditions of model A 


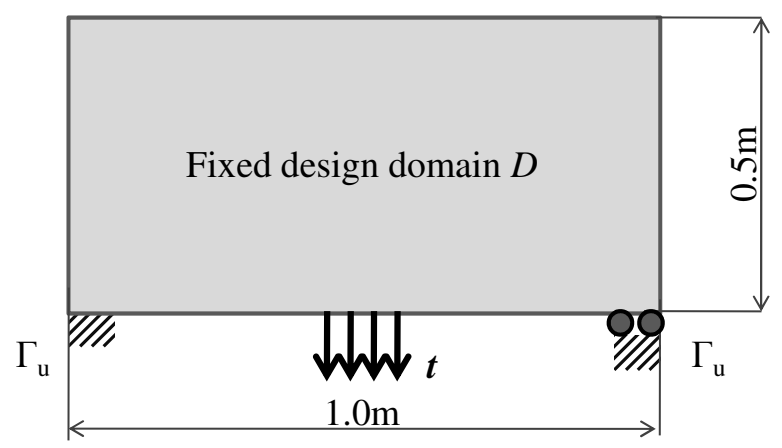

Fig. 3. Fixed design domain and boundary conditions of model B 


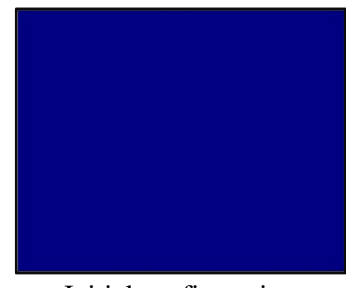

Initial configuration

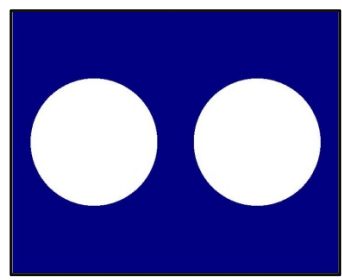

Initial configuration

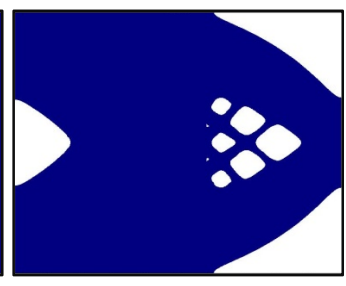

Step 10

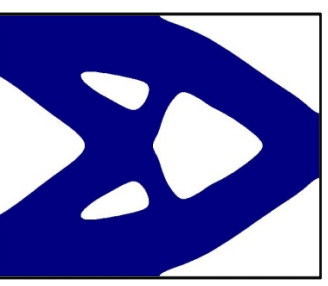

Step 50

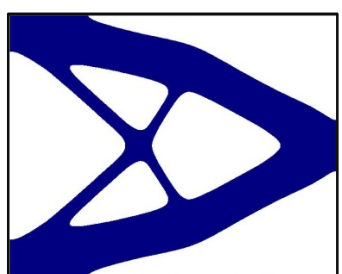

Optimal configuration

(a) Case 1

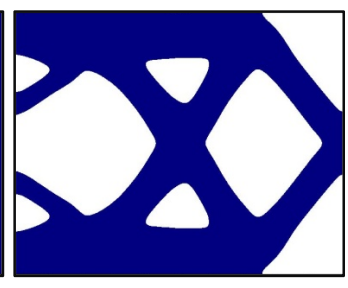

Step 10

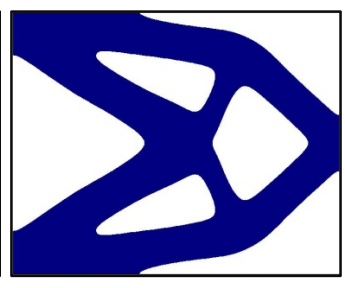

Step 50

(b) Case 2

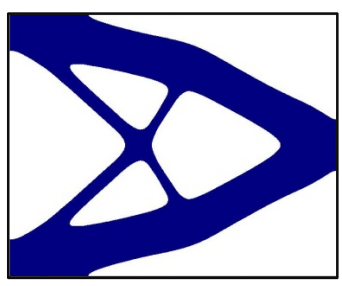

Optimal configuration

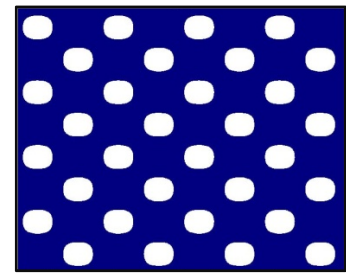

Initial configuration

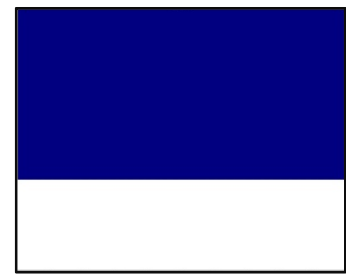

Initial configuration

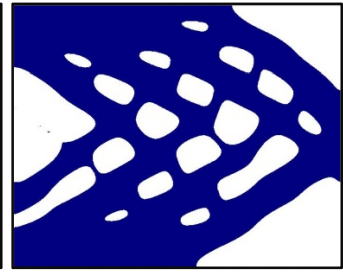

Step 10

(c) Case 3

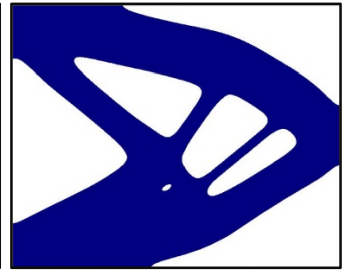

Step 50

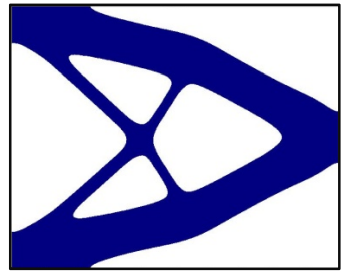

Optimal configuration

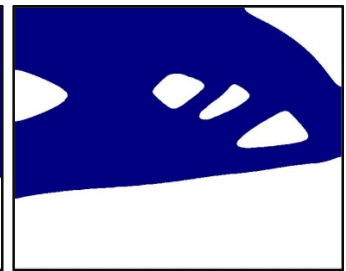

Step 10

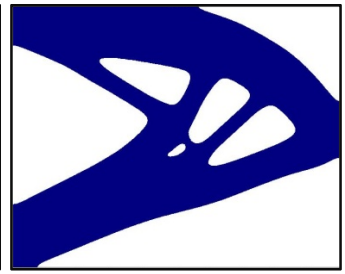

Step 50

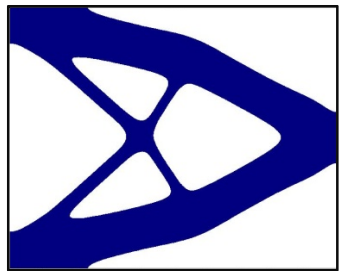

Optimal configuration

(d) Case 4

Fig. 4. Initial configurations, intermediate results and optimal configurations 


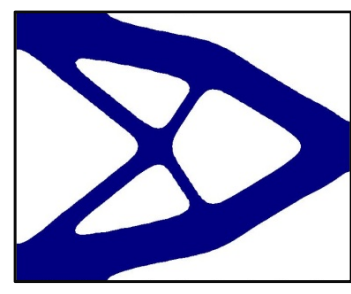

(a) $80 \times 60$ mesh

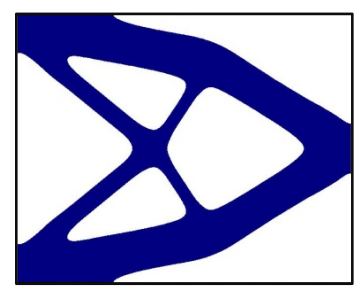

(b) $160 \times 120$ mesh

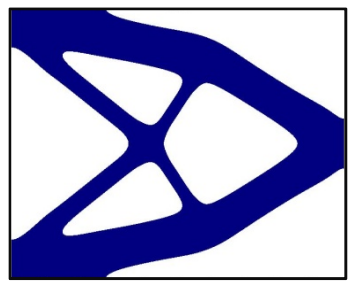

(c) $320 \times 240$ mesh

Fig. 5. Optimal configurations: (a) $80 \times 60$ mesh; (b) $160 \times 120$ mesh; (c) $320 \times 240$ mesh 


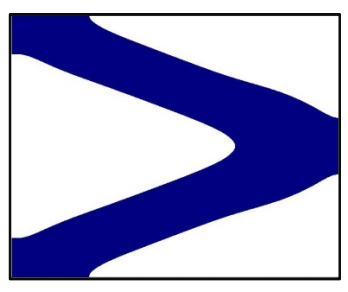

(a) Case 1

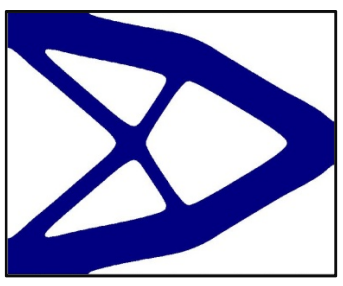

(b) Case 2

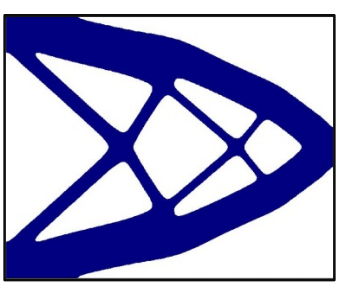

(c) Case 3

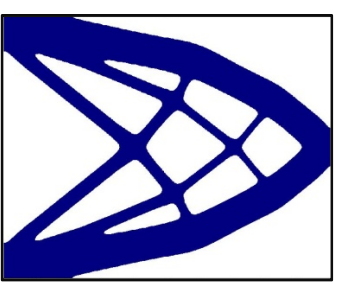

(d) Case 4

Fig. 6. Optimal configurations: (a) $\tau=5 \times 10^{-4}$; (b) $\tau=5 \times 10^{-5}$; (c) $\tau=3 \times 10^{-5}$; (d) $\tau=2 \times 10^{-5}$ 


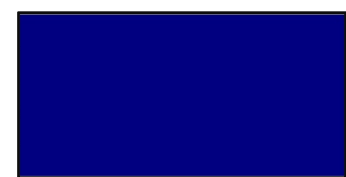

Initial configuration

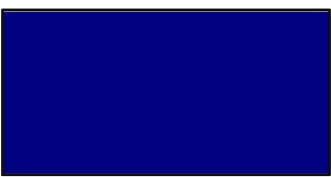

Initial configuration

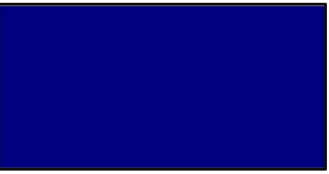

Initial configuration

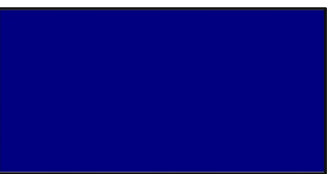

Initial configuration

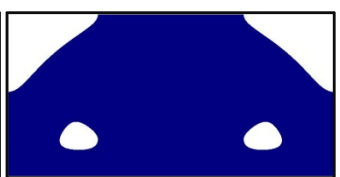

Step 10

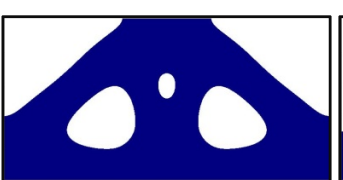

Step 50

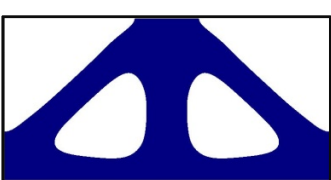

Optimal configuration

(a) Case 1

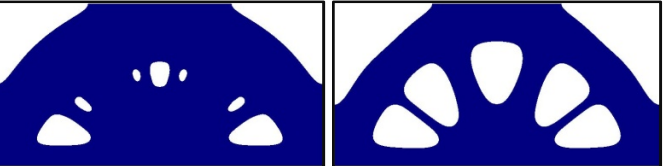

Step 10

Step 50 (b) Case 2

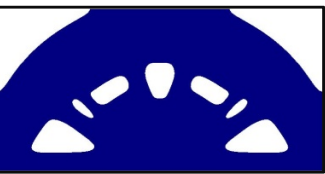

Step 10

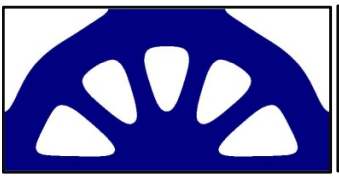

Step 50

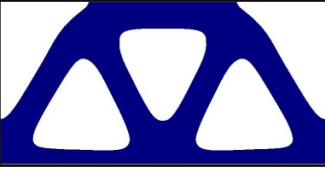

Optimal configuration

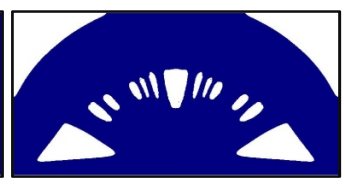

Step 10

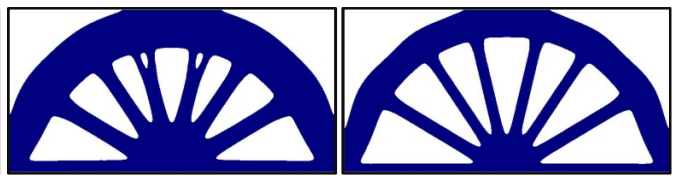

Step 50

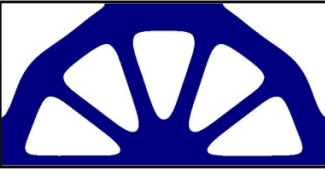

Optimal configuration

(d) Case 4

Fig. 7. Initial configurations, intermediate results and optimal configurations: (a) $\tau=5 \times 10^{-4}$; (b) $\tau=2 \times 10^{-4}$; (c) $\tau=1 \times 10^{-4}$; (d) $\tau=1 \times 10^{-5}$ 


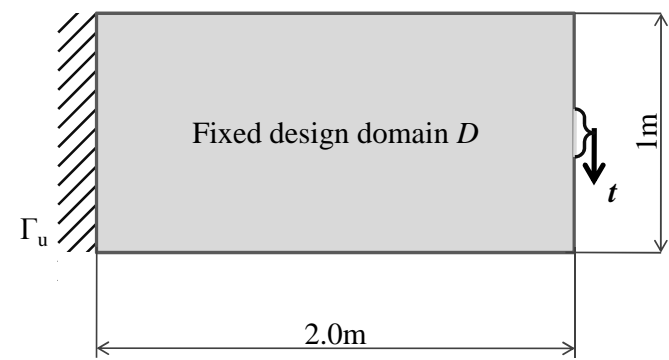

Fig. 8. Fixed design domain and boundary conditions of model $\mathrm{C}$ 


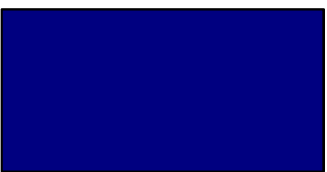

Initial configuration

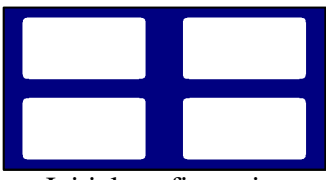

Initial configuration

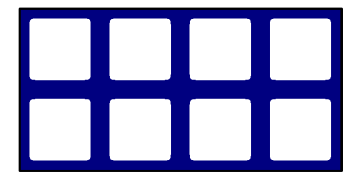

Initial configuration

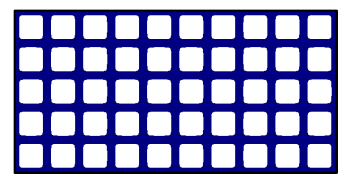

Initial configuration

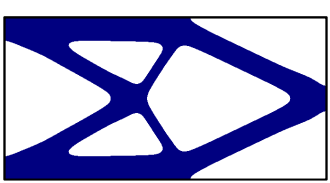

$K(\phi)=K_{\mathrm{cos}}$

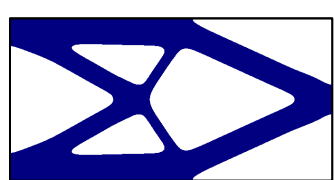

$K(\phi)=K_{\text {sin }}$

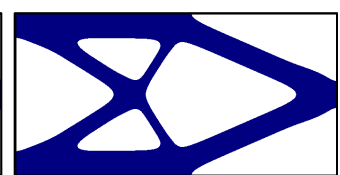

$K(\phi)=K_{1}$

(a) Case 1

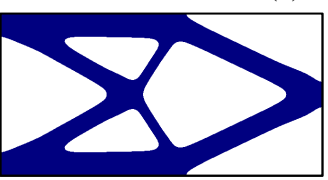

$K(\phi)=K_{\mathrm{cos}}$

(b) Case 2

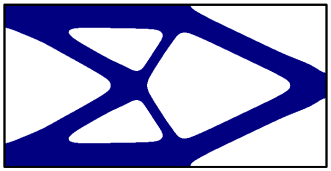

$K(\phi)=K_{\mathrm{cos}}$

(c) Case 3

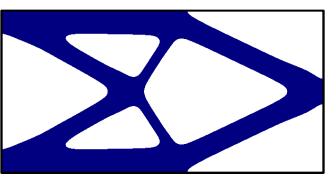

$K(\phi)=K_{\cos }$

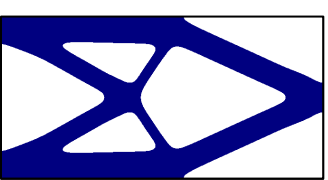

$K(\phi)=K_{\text {si }}$

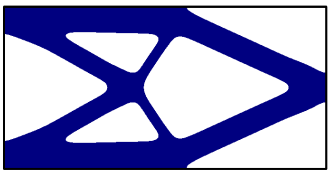

$K(\phi)=K_{\text {sin }}$

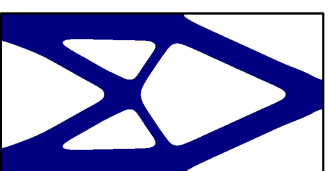

$K(\phi)=K_{\text {sin }}$

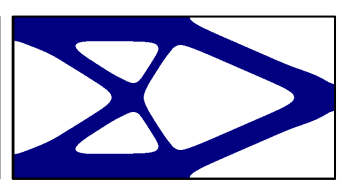

$K(\phi)=K_{1}$

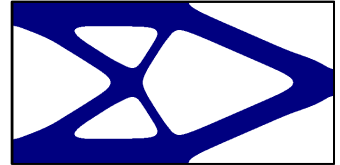

$K(\phi)=K_{1}$

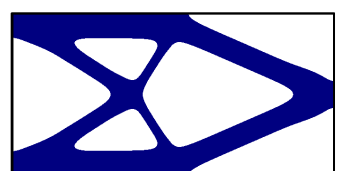

$K(\phi)=K_{1}$

(d) Case 4

Fig. 9. Initial configurations and optimal configurations 


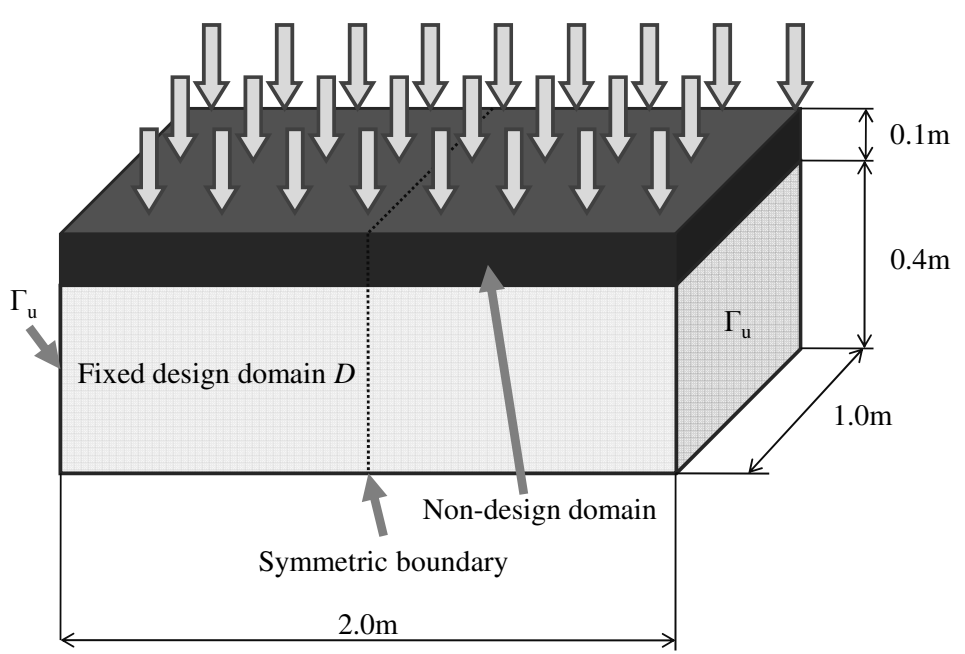

Fig. 10. Fixed design domain and boundary conditions for three dimensional design problem 

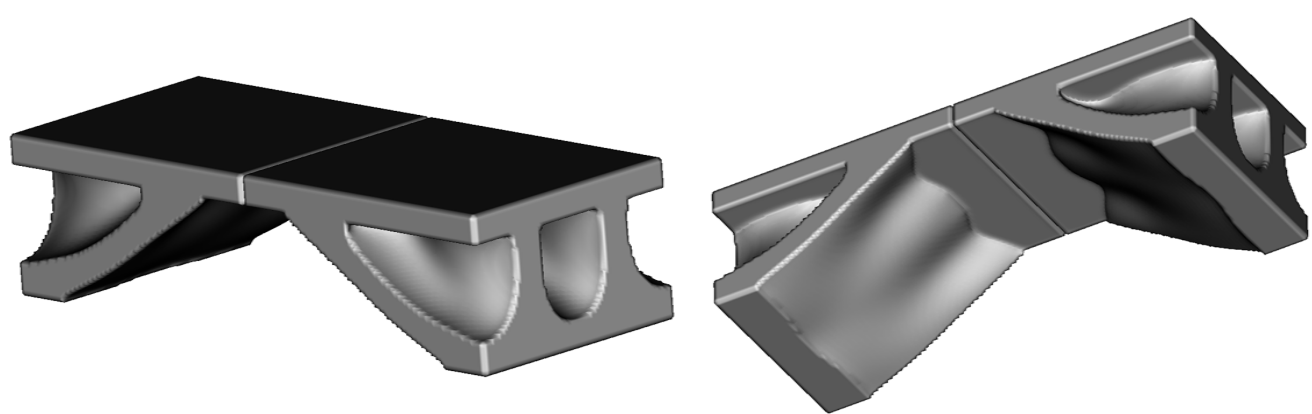

(a) Case 1
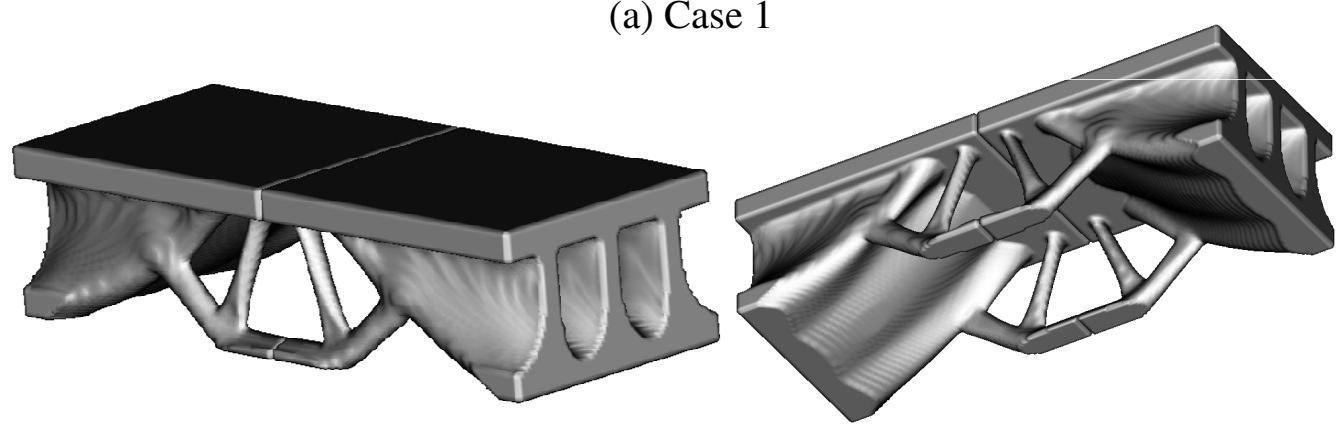

(b) Case 2

Fig. 11. Optimal configurations: (a) $\tau=2 \times 10^{-4}$; (b) $\tau=2 \times 10^{-5}$ 


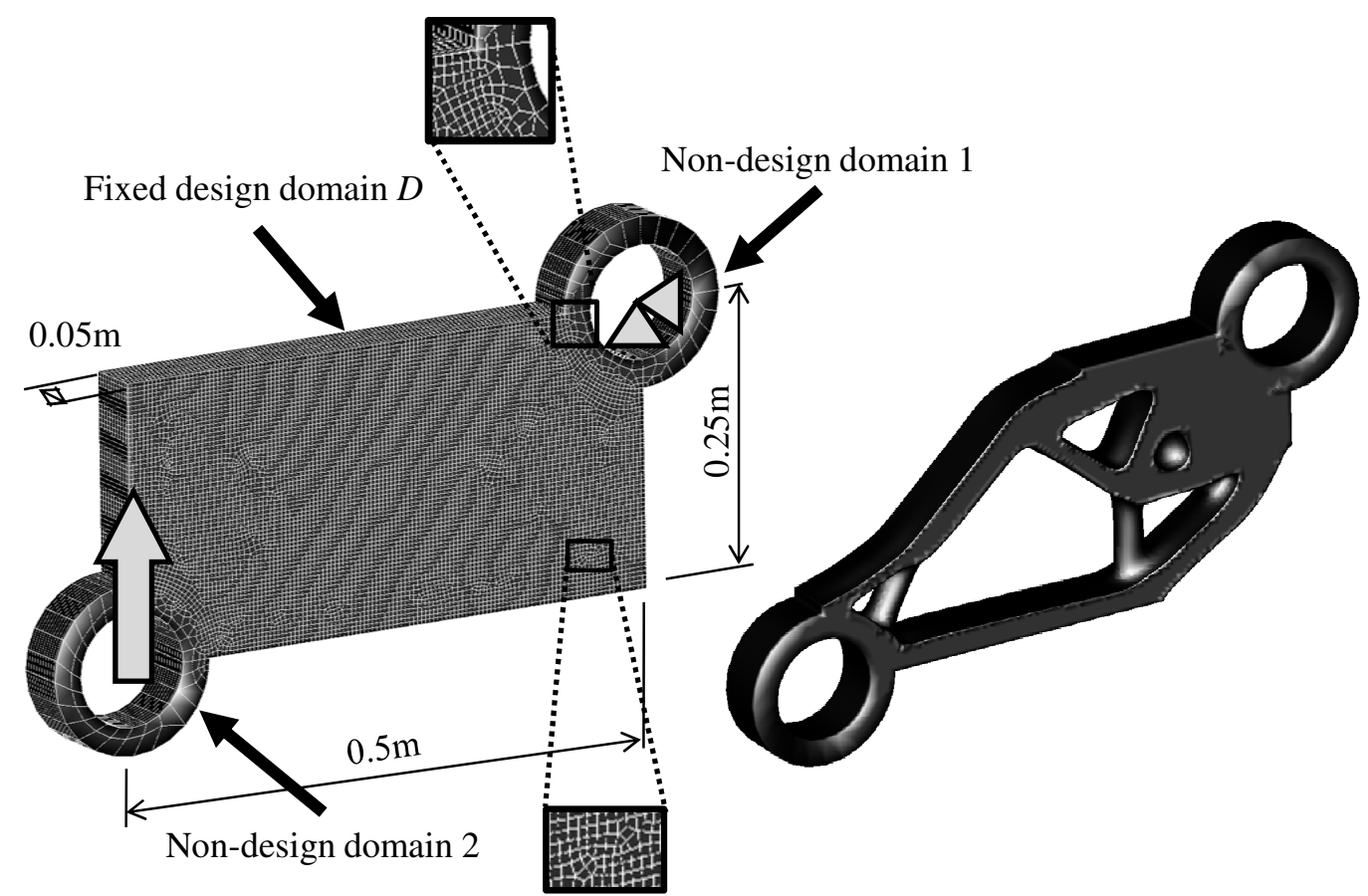

(a) Design domain and boundary conditions

(b) Optimal configuration

Fig. 12. Fixed design domain, boundary conditions and optimal configuration for a mechanical part model 


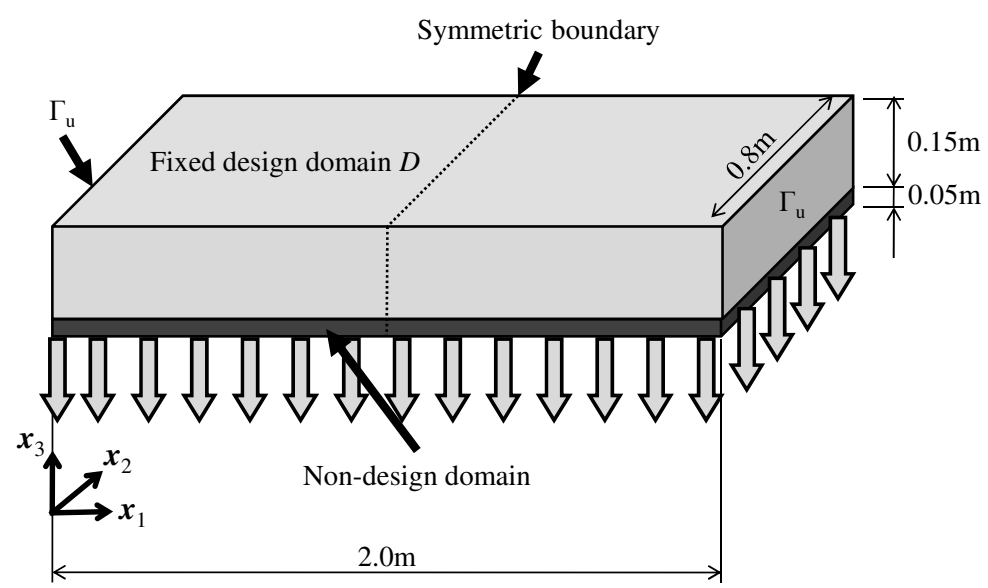

Fig. 13. Fixed design domain and boundary conditions 


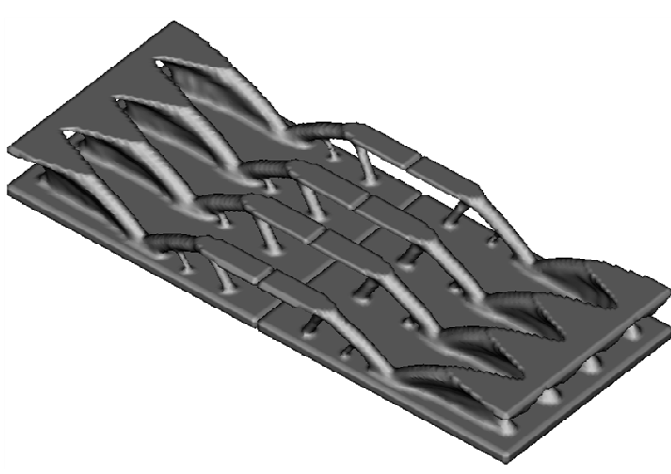

(a) Non-uniform cross-section surface

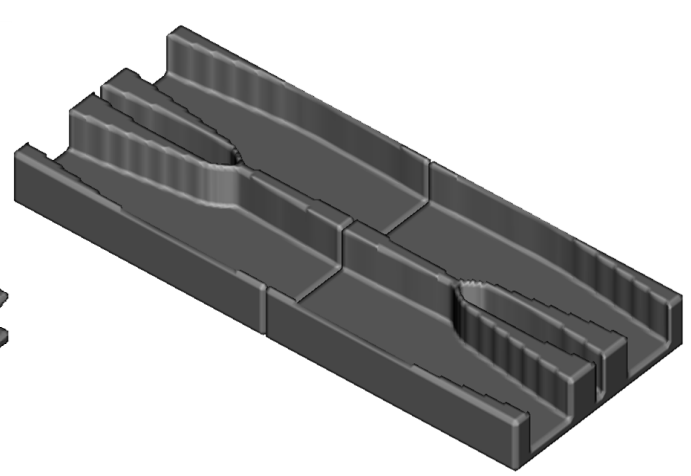

(b) Uniform cross-section surface

Fig. 14. Optimal configurations: (a) Non-uniform cross-section surface; (b) Uniform cross-section surface 


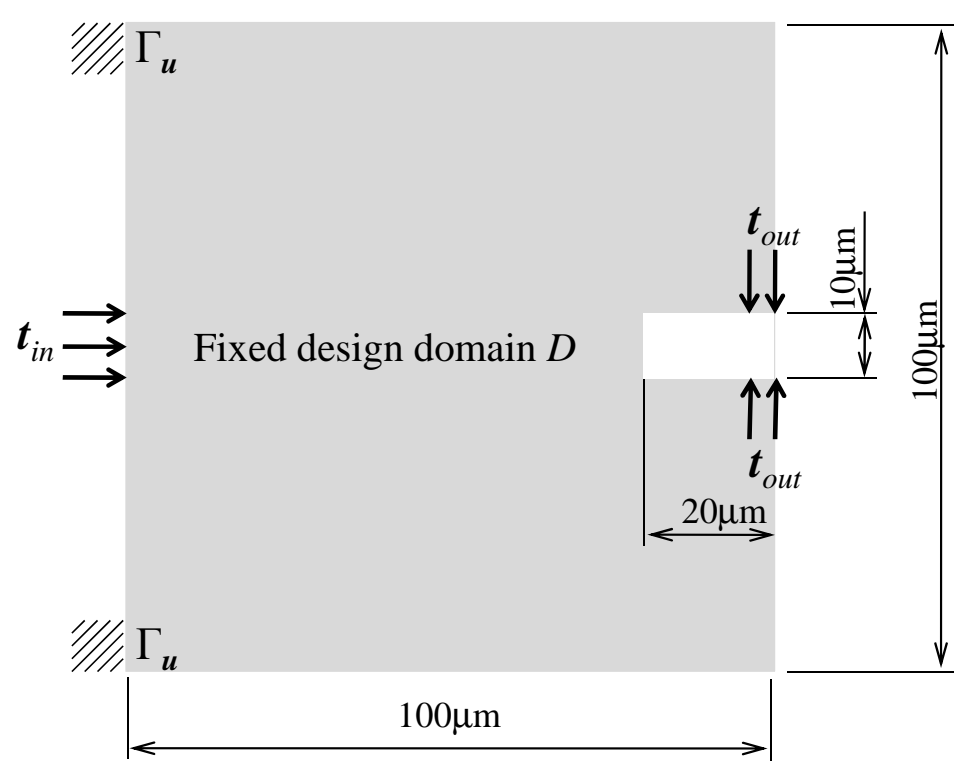

Fig. 15. Fixed design domain for a two-dimensional compliant mechanism 


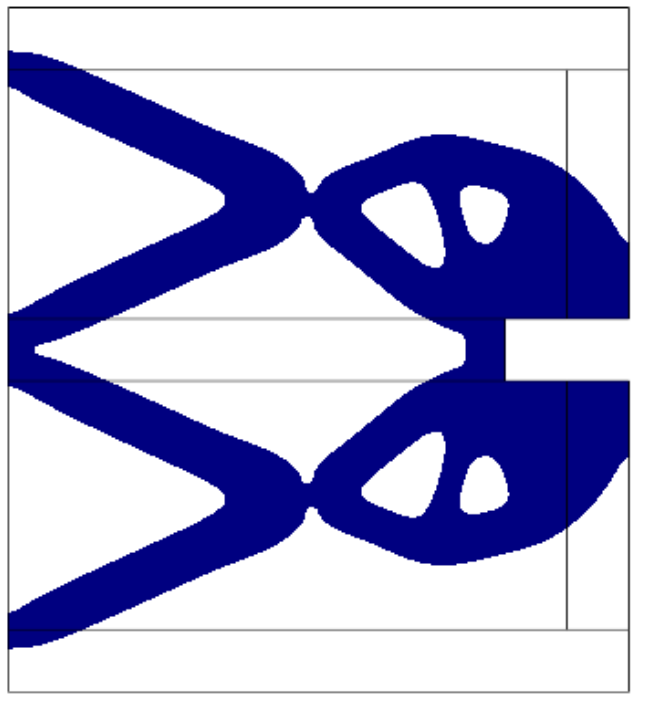

(a) Optimal configuration

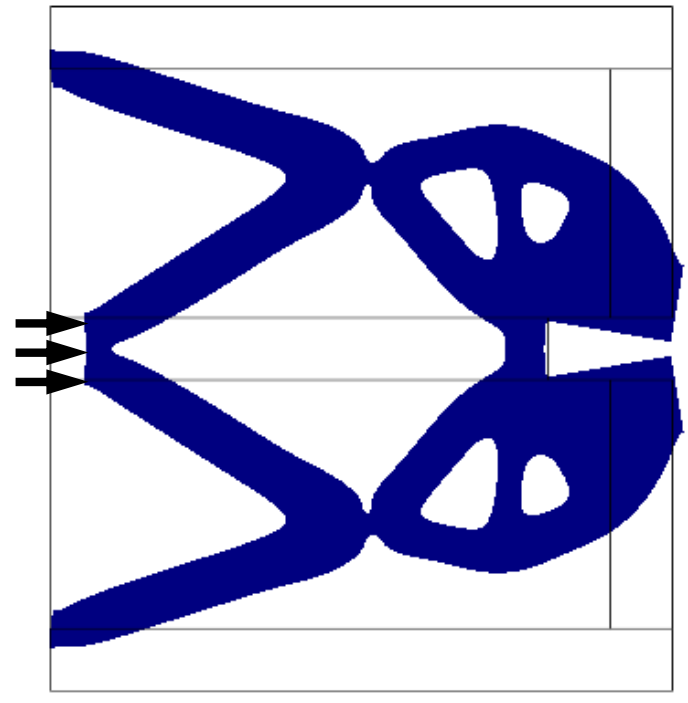

(b) Deformed shape

Fig. 16. Configurations of the two-dimensional compliant mechanism (a) Optimal configuration; (b) Deformed shape 


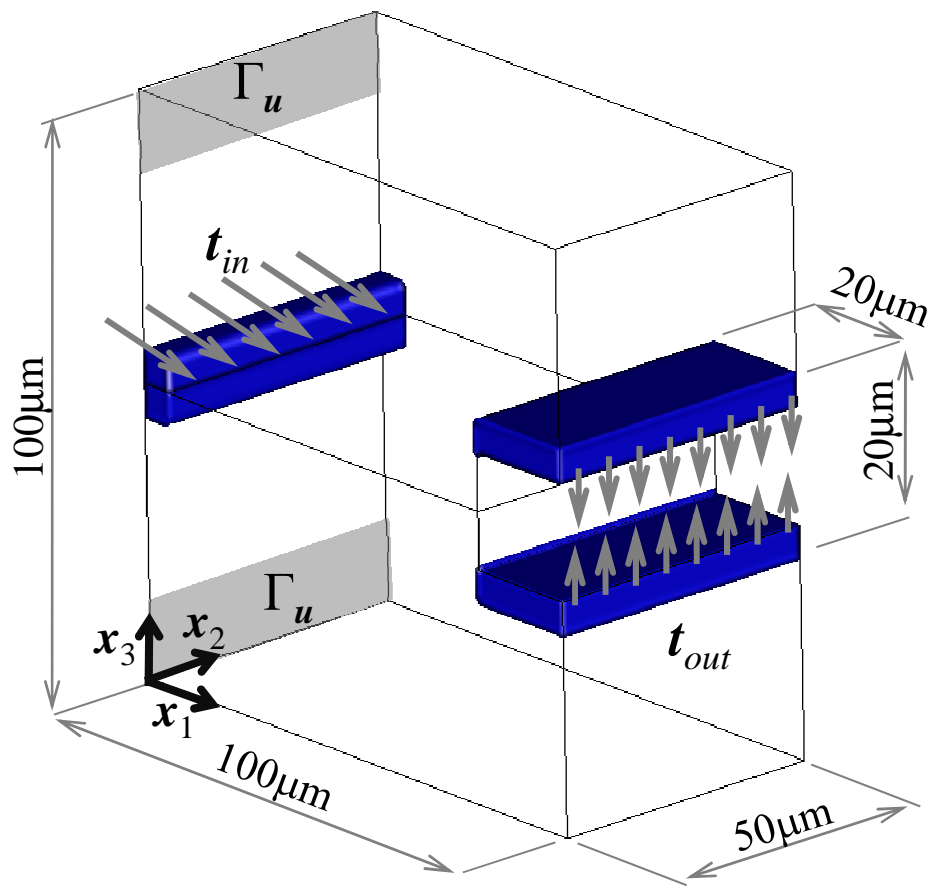

Fig. 17. Fixed design domain for a three-dimensional compliant mechanism 

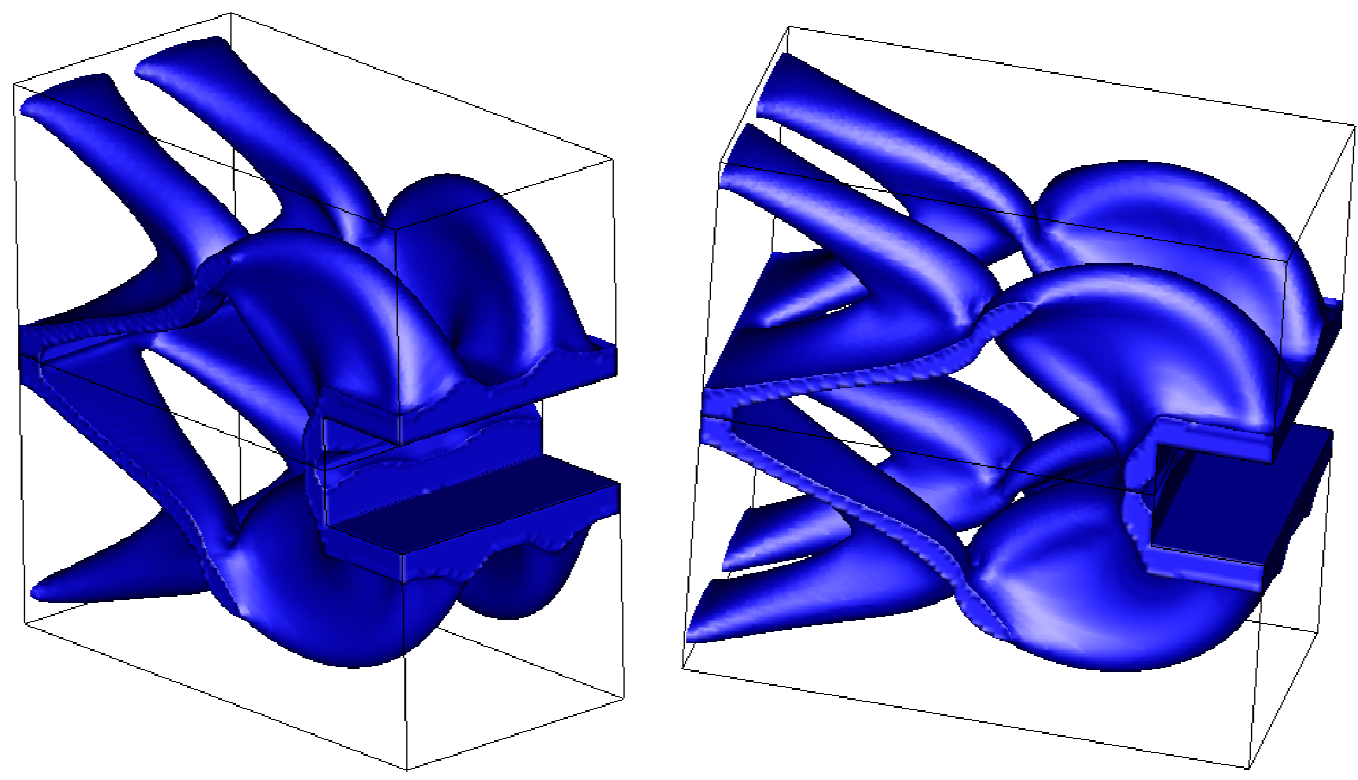

(a) Non-uniform cross-section surface
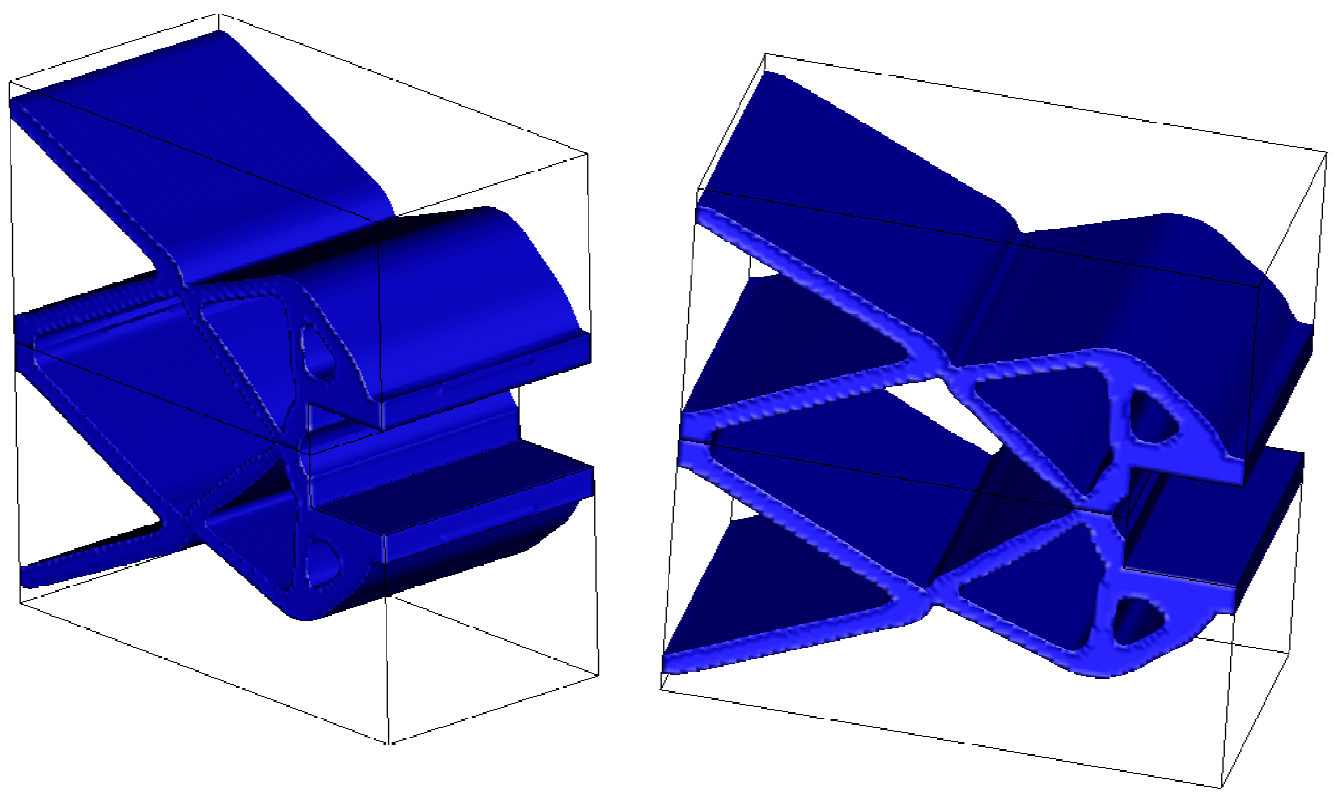

(b) Uniform cross-section surface

Fig. 18. Configurations of the three-dimensional the compliant mechanisms: (a) Non-uniform cross-section surface (b) Uniform cross-section surface 


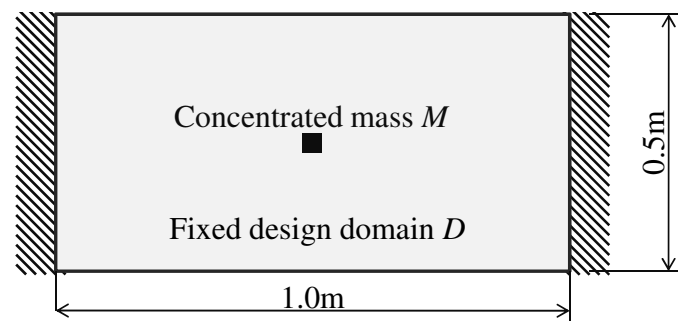

Fig. 19. Fixed design domain for the two-dimensional the lowest eigenfrequency maximization problem 


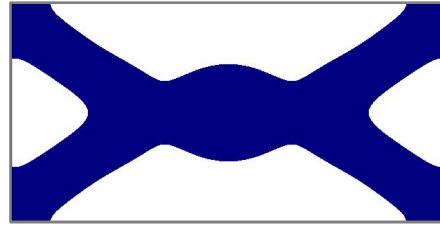

(a) Case 1

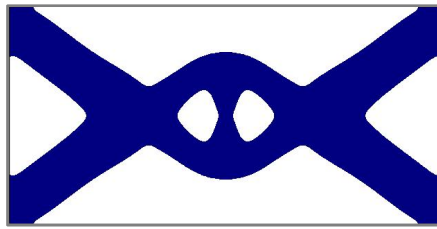

(b) Case 2

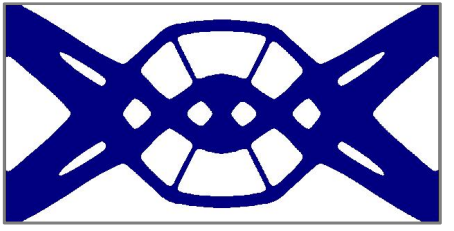

(c) Case 3

Fig. 20. Optimal configurations for the two-dimensional lowest eigenfrequency maximization problem: (a) regularization parameter $\tau=1.0 \times 10^{-4}$; (b) regularization parameter $\tau=1.0 \times 10^{-5}$; (c) regularization parameter $\tau=1.0 \times 10^{-6}$ 


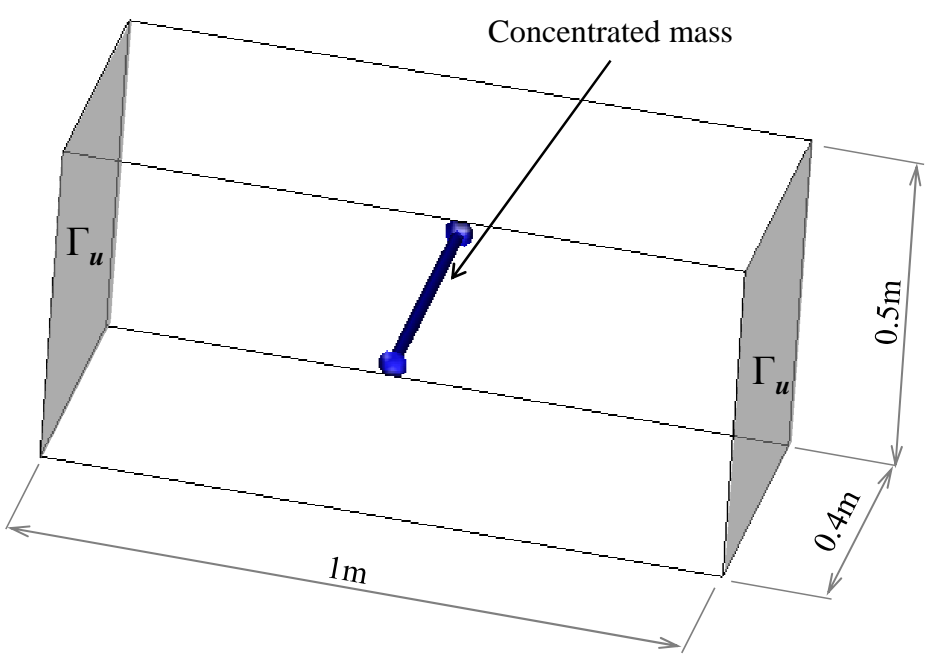

Fig. 21. Fixed design domain for the three-dimensional lowest eigenfrequency maximization problem 

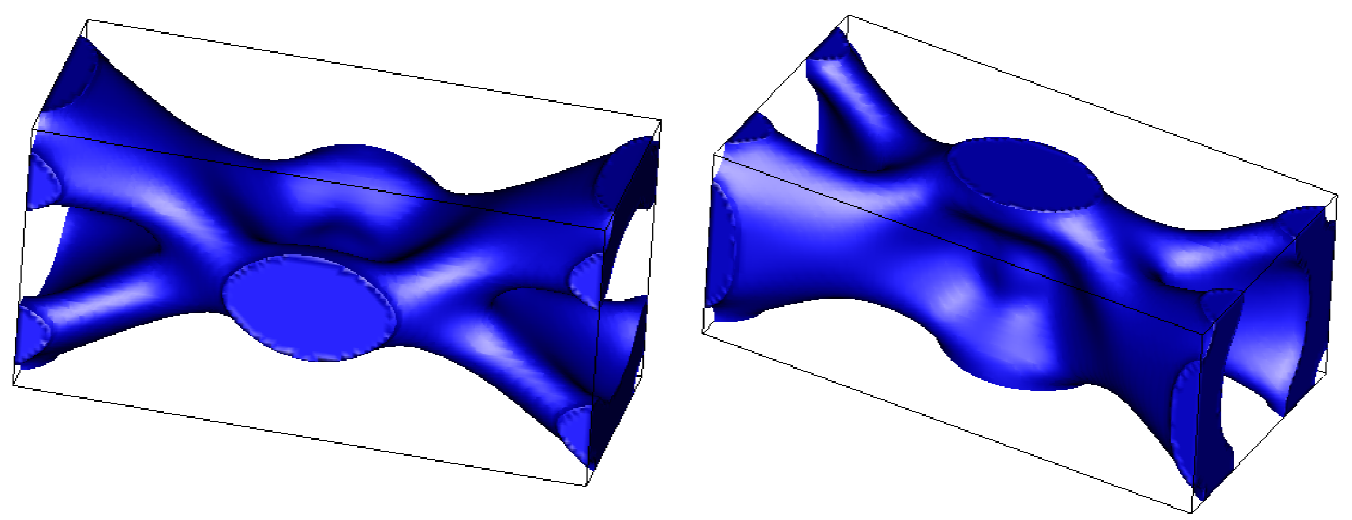

Fig. 22. Optimal configurations of the three-dimensional lowest eigenfrequency maximization problem 